\title{
A Novel Dynamic Multicriteria Decision-Making Approach for Low-Carbon Supplier Selection of Low-Carbon Buildings Based on Interval-Valued Triangular Fuzzy Numbers
}

\author{
Xia Cao, Zeyu Xing (D), Yuqi Sun, and Shi Yin \\ School of Economics and Management, Harbin Engineering University, Harbin, Heilongjiang 150001, China \\ Correspondence should be addressed to Zeyu Xing; hrq962@163.com
}

Received 27 April 2018; Accepted 5 September 2018; Published 24 October 2018

Guest Editor: Tayfun Dede

Copyright ( 2018 Xia Cao et al. This is an open access article distributed under the Creative Commons Attribution License, which permits unrestricted use, distribution, and reproduction in any medium, provided the original work is properly cited.

\begin{abstract}
Due to the lack of natural resources and environmental problems which have been appearing increasingly, low-carbon buildings are more and more involved in the construction industry. The selection of low-carbon supplier is a significant part in the process of low-carbon building construction projects. In this paper, we propose a novel dynamic multicriteria decision-making approach for low-carbon supplier selection in the process of low-carbon building construction projects to deal with these problems. First, the paper establishes 5 main criteria and 17 subcriteria for low-carbon supplier selection in the process of low-carbon building construction projects. Then, a method considering interaction between criteria and the influence of constructors subjective preference and objective criteria information is proposed. It uses the basic concept and properties of the interval-valued triangular fuzzy number intuitionistic fuzzy weighted Bonferroni means (IVTFNIFWBM) operators and the objective information entropy and TOPSIS-based Euclidean distance to calculate the comprehensive evaluation results of potential low-carbon suppliers. The proposed method is much easier for constructors to select low-carbon supplier and make the localization of low-carbon supplier more practical and accurate in the process of building construction projects. Finally, a case study about a low-carbon building project is given to verify practicality and effectiveness of the proposed approach.
\end{abstract}

\section{Introduction}

Low-carbon building has been a popular research topic from academic and industrial sectors in recent years. Buildings play a central part in causing greenhouse gas (GHG) emissions and account for nearly $70 \%$ of GHG emissions in Hong Kong and up to $40 \%$ of total energy consumption [1]. These facts show that low-carbon building plays an important role in reducing the amount of GHG emissions. Many countries have launched a series of measures to reduce GHG emissions in the construction industry [2]. To cope with pressure, it is a vital factor to select their suitable lowcarbon suppliers. Many factors should be taken into account in the process of low-carbon supplier selection as a complex multicriteria decision-making (MCDM) problem [3]. Therefore, it is critically important and necessary to study low-carbon supplier selection in the process of low-carbon building construction projects.
Many scholars have stressed the importance of selecting suitable criteria in the process of low-carbon supplier selection. Lee et al. [4] proposed 5 main criteria for supplier selection, such as quality, technology capability, pollution control, green products, and green competencies. Hsu et al. [5] established 13 criteria of supplier selection with three main criteria, such as planning, implementation, and management. Kannan et al. [6] and Tsui and Wen [7] thought low-carbon supplier selection should consider lowcarbon criteria in environmental aspects, such as waste reduction, green technologies, and the usage of ecodesign. Gurel et al. [8] established 8 main criteria that include cost, delivery, quality, service, strategic alliance, and pollution control. Chen et al. [9] proposed 20 criteria for supplier selection and evaluation criteria with two dimensions (economic criteria and environmental criteria). Yu et al. [10] took the economic criteria and environmental criteria into consideration during low-carbon supplier selection. 
Govindan and Sivakumar [11] took economics, operational factors, and environmental criteria into consideration. Pang et al. [12] proposed 4 main criteria including production and service. However, most of them focus on low-carbon supply chain management, and the research on the low-carbon supplier selection in the process of low-carbon building construction projects is fairly rare. Moreover, comparing with the traditional low-carbon supplier selection criteria, constructors must pay special attention to the environmental capabilities, low-carbon building technologies, and social factors for low-carbon supplier selection criteria in the process of low-carbon building construction projects [13]. This research takes these aspects into consideration, which have been ignored in many studies such as an evaluation criterion.

In recent years, extensive MADM methods have been proposed for supplier selection. Govindan et al. [14] concluded that the most frequently used method is AHP (27.78\%), followed by ANP (16.6\%), DEA (11.1\%), LP $(8.76 \%)$, TOPSIS $(5.56 \%)$, and multiobjective optimization $(2.77 \%)$. In addition, many methods have been developed to select suitable low-carbon supplier based on specific methods that include fuzzy set theory [9, 12, 15, 16, 19], genetic algorithm [17-19], structural equation modeling and fuzzy logic [20], and artificial neural network [21, 22]. Hu et al. [23] proposed a multicriteria group decision-making method with 2-tuple linguistic assessments for low-carbon supplier selection under a fuzzy uncertain information environment. Qin et al. [24] developed a new TODIM technique to select low-carbon supplier within the context of interval type-2 fuzzy sets. Bakeshlou et al. [25] presented a multiobjective hybrid fuzzy linear programming model for low-carbon supplier selection problem.

However, most of these methods, which do not consider interaction between criteria, can lead to irrational decisionmaking of low-carbon supplier selection in the process of low-carbon building construction projects. In fact, there is always an interactive relationship between criteria of lowcarbon supplier selection, such as complementarity between criteria, the redundancy of criteria, and preference relation of criteria.

The Bonferroni mean (BM) is a mean type aggregation technique, which considers interaction between attributes that makes it very useful in decision-making [26, 27]. Then, many scholars proposed BM operator [26, 27], IFBM operator [27, 28], IFGBM operator [26, 29, 30], and WIFBM operator [31,32]. Unfortunately, there still is a lack of further theory and method research on the TFNIFN based on BM operator. Therefore, this paper focuses on a dynamic multiattribute decision-making method with interval-valued triangular fuzzy number intuitionistic fuzzy that considers interaction between attributes.

In real life, past and current information should also be considered when conducting dynamic decision-making, and how to solve the problem of time sequences weight has become the key to solving the dynamic decision-making problem. Scholars, such as Wei [33], Park et al. [34], and Yin et al. [35], have designed dynamic intuitionistic fuzzy decision models of time dimension. At present, some of the commonly used time sequence weights are as follows: the arithmetic progression and geometric progression method [36], the binomial distribution method [37], the normal distribution method [38], the exponential distribution method [39], and the time sequence ideal solution method [40, 41]. These methods provide a reference for solving the time sequence weights in dynamic multiattribute decision-making problems, but their weights fully based on objective assignment methods or decision maker's subjective preference, and did not consider to combine objective assignment methods with decision maker's subjective preference. In our paper, we construct a comprehensive time weight while considering the objective assignment information as well as subjective preference. In addition, dynamic stochastic multiattribute decision-making problems possess a time dimension and an attribute dimension, so determining attribute weights is a prerequisite for assembling the attribute information required for the final decision-making result. Relevant scholars have developed a variety of methods for successfully determining attribute weight, Wei [42] has designed a new method based on maximizing deviation and two-tuple, Chen et al. [43] have obtained attribute weights by solving the grey relation function of attribute information per the grey correlation model, and Wang et al. [44] have proposed a method by using hesitant fuzzy entropy. Finally, we provide a new method of calculating the attribute weight by objective information entropy and TOPSIS-based Euclidean distance.

The main contribution of this paper is developing a new dynamic MADM that considers interaction between criteria under time sequence for low-carbon supplier selection in the process of low-carbon building construction projects. The new dynamic multiattribute decision-making method is proposed with the interval-valued triangular fuzzy number intuitionistic fuzzy weighted Bonferroni means (IVTFNIFWBM) operator that considers interaction between attributes under time-sequence. This method puts forward some concepts of IVTFNIFWBM operator and proves that. To calculate attribute weights, we introduce the objective information entropy and TOPSIS-based Euclidean distance and present a new weight calculation method of IVTFNIFWBM. Also, the method constructs a comprehensive time weight while considering the objective assignment information as well as subjective preference and can reflect the process of dynamic decision-making more comprehensively and reasonable. The proposed method has been successfully implemented in case construction projects to select the best low-carbon supplier. Besides, the developed method can be widely used as a structural model for lowcarbon supplier selection in other industries.

The structure of this paper is organized as follows. The proposed methodological framework for low-carbon supplier evaluation and selection is presented in Section 2. Section 3 establishes the criteria for low-carbon supplier selection in the process of low-carbon building construction projects. Section 4 draws some related concepts of the proposed approach for low-carbon supplier selection. Section 5 proposes a method that considers interaction between criteria under time sequence based on IVTFNIFWBM operator and comprehensive time sequence weighted 
calculation model and a new method of attribute weighted based on the objective information entropy and TOPSISbased Euclidean distance for low-carbon supplier selection. Section 6 provides a real case study that concerns lowcarbon supplier selection in the process of low-carbon building construction projects. In Section 7, we end the paper by summarizing the conclusions.

\section{Methodological Framework for Low-Carbon Supplier Evaluation and Selection}

The proposed framework for low-carbon supplier evaluation and selection of low-carbon buildings is illustrated in Figure 1, and it mainly consists of three stages. First, the low-carbon supplier selection criteria in the process of low-carbon building construction projects are identified from the comprehensive literature review, on-site investigation, and the policy analysis according to the triple bottom line principle. Various realistic features in supplier selection of low-carbon building construction projects are considered. Second, the validity of low-carbon supplier selection criteria is assessed by senior purchasing experts and project managers with rich civil industry experience, and then, we further modify the lowcarbon supplier selection criteria until the validity of criteria is satisfactory according to the feedbacks of experts and project managers. Then the experts and project managers evaluate alternative low-carbon supplier. The best alternative is selected via the interval-valued triangular fuzzy multicriteria decision-making model, which is mainly made up of four procedures, including calculating attribute weight based on Entropy-TOPSIS, calculating time weight based on time degree and ideal solution, calculating information contents by IVTFIFWBM operator, and evaluating and selecting the best low-carbon supplier. These procedures of the fuzzy multicriteria decision-making model will be introduced in Section 5 in detail.

\section{Low-Carbon Supplier Evaluation Criteria}

For most projects, in the process of low-carbon building construction, the successful implementation of a project requires selecting low-carbon supplier that contributes to the project objective. Low-carbon supply chain in the construction industry is a functional network structure model, which consists of main parts of construction industry, with building units as the core and logistics, capital flow, information flow, and knowledge flow as the support in the whole life cycle of building projects. In this section, we will introduce the proposed criteria for low-carbon supplier selection based on above reviews and the identified criteria. We establish 5 main criteria and 17 subcriteria for low-carbon supplier selection in the process of low-carbon building construction projects (Table 1).

Low-carbon materials information is the basic point for low-carbon supplier selection in the process of low-carbon building construction projects. In building's construction process, projects demand different product types, such as different types of concrete, steel, and templet, and product structure to guarantee the successful completion of construction projects. Therefore, low-carbon supplier selection in the process of low-carbon building construction projects should focus on materials flexibility, efficiency, information, and other aspects of building materials. It is particularly important to provide constructors with high quality and inexpensive building materials or service, such as payment terms, to meet the needs of constructor. In addition, the low-carbon degree of building materials reflects its ability of saving resources and reducing energy consumption, and the higher the low-carbon degree is, the more application potential the building materials will have in the future. Meanwhile, it also needs to improve service quality and user experience and strengthens after sales service support. Therefore, building materials information is mainly reflected from four aspects: materials cost, low-carbon degree of materials, materials quality, and materials flexibility.

In the complex and changing market environment, the competitiveness of low-carbon supply chain in the construction industry depends on rapid response to the needs of different product types and product structure in building's construction process. High level of low-carbon business operation can contribute to reducing carbon emissions which can be reflected by the level of low-carbon information sharing, the cost control of transportation, and the supply chain management of construction industry. In addition, constructors need to consider the financial capability to reduce the risk of cooperation between constructors and its suppliers' protection for the successful completion of construction projects. Here, we use level of low-carbon information sharing, low-carbon logistics, financial capability, and emergency response capability to measure the supplier's low-carbon business operation.

In the construction industry, the main purpose of establishing low-carbon supply chain is to establish cooperative alliance of construction industry, which can reduce building materials' cost and obtain more income in projects. Cooperation potential is the premise of establishing strategic alliance, and strong cooperation intention and long-time cooperation are the foundation of establishing strategic alliance. If constructors want to maintain the long-term stability of low-carbon supply chain cooperation, they should choose those suppliers who have advanced management and desire of low-carbon cooperation for development. We can measure potential for sustainable cooperation from these four aspects: compatibility of lowcarbon culture, desire of low-carbon cooperation, enterprise reputation, and low-carbon image.

Low-carbon culture can promote the implementation of enterprise strategic objectives of sustainable development virtually. If the low-carbon culture between partners cannot be integrated, it means that it will lead to different values between constructors and suppliers. Then, it may lead to dispute on both sides of the fierce confrontation and even relationship broken. Ecodesign of building materials can reduce environmental pollution in the production process and reduce carbon emissions. In addition, low-carbon certifications reflect the environmental management capability of low-carbon supplier.

Low-carbon technology capability, which is used to evaluate whether the low-carbon supplier meets the 


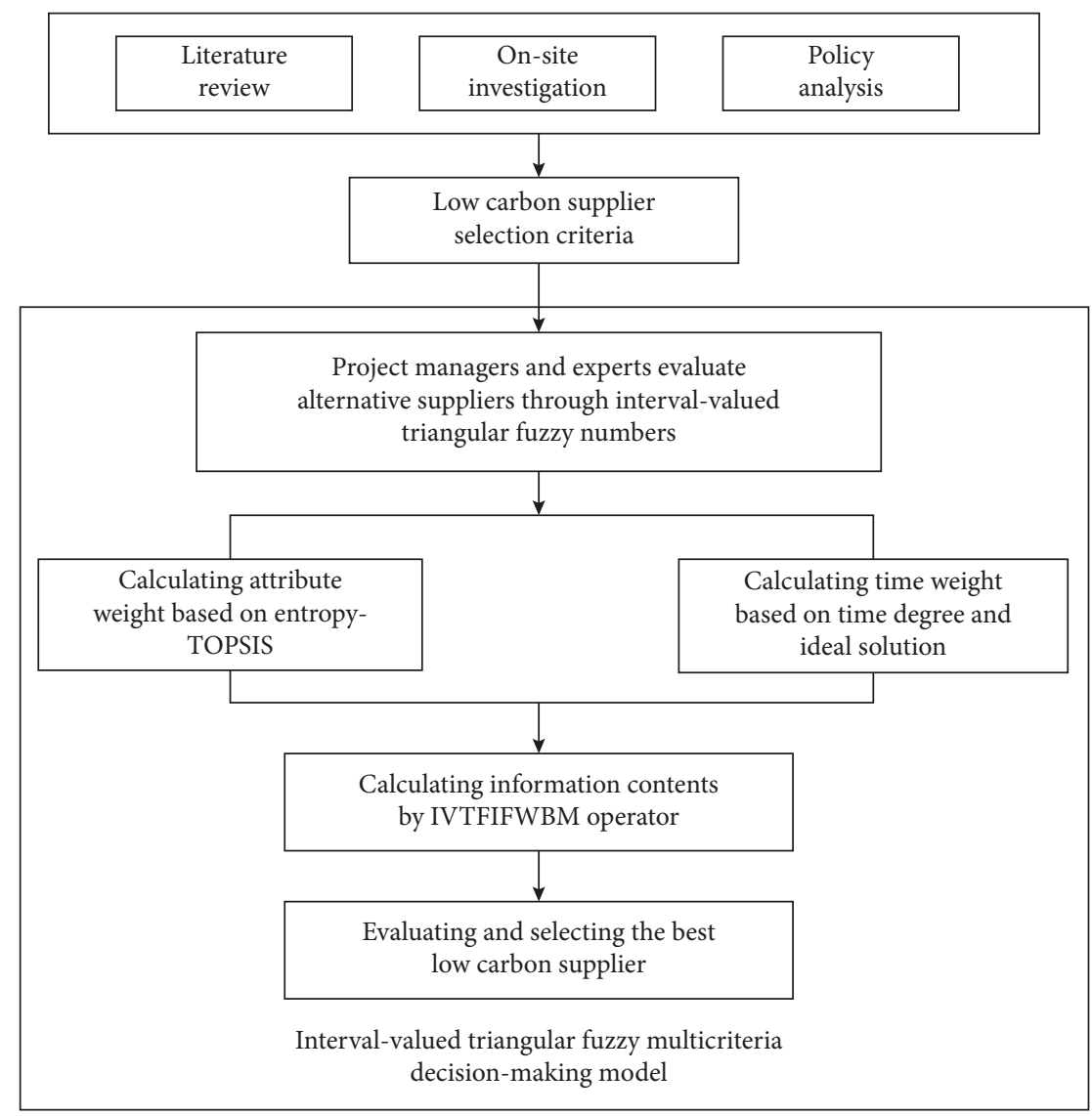

FIGURE 1: Methodological framework for low-carbon supplier evaluation and selection.

TABLE 1: Criteria for low-carbon supplier selection in the process of low-carbon building construction projects.

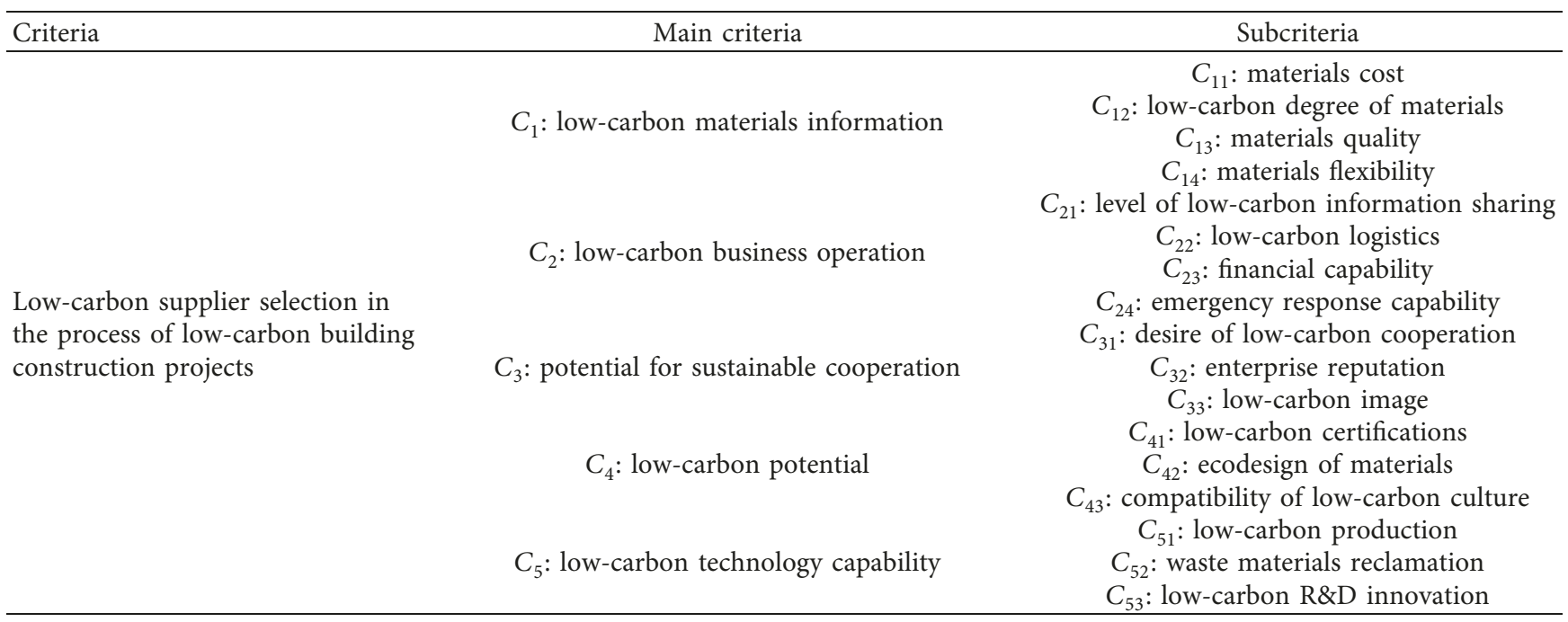

requirements of low-carbon building, is increasingly crucial to successfully implement low-carbon building and attain sustainability goals. Low-carbon building technologies are incorporated into building design and construction to make the end product sustainable. Low-carbon R\&D innovation include new launch of building materials and low-carbon building technologies, and there are many different low-carbon building technologies applicable in the whole process of delivering building projects.

\section{Preliminaries}

Here, we introduce some basic concepts and terminologies of intuitionistic fuzzy set (IFS), which will be used in the proposed method. Its definition is introduced as follows: 
Definition 1. Let $A$ as an intuitionistic fuzzy set (IFS), and $A=\left\{\left\langle x, u_{A}(x), v_{A}(x)\right\rangle\right\} x \in X$ with the condition that $[45]$

$$
\begin{aligned}
u_{A}: X & \longrightarrow[0,1], x \in X \longrightarrow u_{A}(x) \in[0,1] \\
v_{A}: X & \longrightarrow[0,1], x \in X \longrightarrow u_{A}(x) \in[0,1], \\
& 0 \leq u_{A}(x)+v_{A}(x) \leq 1, x \in X .
\end{aligned}
$$

We can find that an IFS is constructed by two information functions, which not only describe the membership degree $u_{A}(x)$, but also describe the nonmembership degree $v_{A}(x)$. Moreover, the hesitancy information of $x \in X$ can be denoted by $\pi_{A}(x)=1-$ $u_{A}(x)-v_{A}(x)$ which is called the hesitant index, and therefore IFS can describe the uncertainty and fuzziness more objectively than the usual fuzzy set.

Definition 2. Zadeh first proposed the concept of triangular fuzzy number [43]. Let $X$ as a nonempty finite set. A triangular fuzzy number intuitionistic fuzzy set (TFNIFS) $A$ in $X$ is defined as $X=\left\{\left\langle x, \widehat{u}_{A}(x), \widehat{v}_{A}(x)\right\rangle \mid x \in X\right\}$, where $\widehat{u}_{A}=$ $\left[\widehat{u}_{A}^{l}(x), \widehat{u}_{A}^{m}(x), \widehat{u}_{A}^{u}(x)\right]$ and $\widehat{v}_{A}=\left[\widehat{v}_{A}^{l}(x), \widehat{v}_{A}^{m}(x), \widehat{v}_{A}^{u}(x)\right] \mathrm{de}-$ note, respectively, membership and nonmembership of the element $x$ in $X$ to $A$, and

$$
0 \leq \widehat{u}_{A}^{u}(x)+\widehat{v}_{A}^{u}(x) \leq 1, \widehat{u}_{A}^{l}(x) \geq 0, \widehat{v}_{A}^{l}(x) \geq 0 .
$$

Then, we call $\left(\left[\widehat{u}_{A}^{l}(x), \widehat{u}_{A}^{m}(x), \widehat{u}_{A}^{u}(x)\right],\left[\widehat{v}_{A}^{l}(x), \widehat{v}_{A}^{m}(x)\right.\right.$, $\left.\left.\widehat{v}_{A}^{u}(x)\right]\right)$ as an IVTFNIFN, and it is also called as ([a, $b, c],[d, e, f])[46]$.

Definition 3. Let $\tilde{\alpha}_{1}=\left(\left[a_{1}, b_{1}, c_{1}\right],\left[d_{1}, e_{1}, f_{1}\right]\right)$ and $\widetilde{\alpha}_{2}=$ $\left(\left[a_{2}, b_{2}, c_{2}\right],\left[d_{2}, e_{2}, f_{2}\right]\right)$ are two random IVTFNIFN, then

$$
\begin{aligned}
\tilde{\alpha}_{1} \oplus \tilde{\alpha}_{2}= & \left(\left[a_{1}+a_{2}-a_{1} a_{2}, b_{1}+b_{2}-b_{1} b_{2}, c_{1}+c_{2}-c_{1} c_{2}\right],\right. \\
& {\left.\left[d_{1} d_{2}, e_{1} e_{2}, f_{1} f_{2}\right]\right), }
\end{aligned}
$$

$$
\begin{aligned}
\widetilde{\alpha}_{1} \otimes \widetilde{\alpha}_{2}= & \left(\left[a_{1} a_{2}, b_{1} b_{2}, c_{1} c_{2}\right],\left[d_{1}+d_{2}-d_{1} d_{2}, e_{1}+e_{2}-e_{1} e_{2},\right.\right. \\
& \left.\left.f_{1}+f_{2}-f_{1} f_{2}\right]\right),
\end{aligned}
$$

$$
\begin{aligned}
\lambda \widetilde{\alpha}_{1}= & \left(\left[1-\left(1-a_{1}\right)^{\lambda}, 1-\left(1-b_{1}\right)^{\lambda}, 1-\left(1-c_{1}\right)^{\lambda}\right],\right. \\
& {\left.\left[d_{1}^{\lambda}, e_{1}^{\lambda}, f_{1}^{\lambda}\right]\right), } \\
\widetilde{\alpha}_{1}^{\lambda}= & \left(\left[a_{1}^{\lambda}, b_{1}^{\lambda}, c_{1}^{\lambda}\right],\left[1-\left(1-d_{1}\right)^{\lambda}, 1-\left(1-e_{1}\right)^{\lambda},\right.\right. \\
& \left.\left.1-\left(1-f_{1}\right)^{\lambda}\right]\right) .
\end{aligned}
$$

Definition 4. For any IVTFNIFN $\widetilde{\alpha}=([a, b, c],[d, e, f])$, the score of $\widetilde{\alpha}$ can be evaluated by the score function $S$ as follows [47]:

$$
S(\widetilde{\alpha})=\frac{a+2 b+c}{4}-\frac{d+2 e+f}{4}
$$

where $S(\widetilde{\alpha}) \in[-1,1]$.

And, an accuracy function is shown below:

$$
H(\widetilde{\alpha})=\frac{a+2 b+c}{4}\left(2-\frac{a+2 b+c}{4}-\frac{d+2 e+f}{4}\right) .
$$

Definition 5. Suppose $\widetilde{\alpha}_{1}$ and $\widetilde{\alpha}_{2}$ are two IVTFNIFN [48], where

(1) If $S\left(\widetilde{\alpha}_{1}\right) \prec S\left(\widetilde{\alpha}_{2}\right)$, then $\widetilde{\alpha}_{1} \prec \widetilde{\alpha}_{2}$

(2) If $S\left(\widetilde{\alpha}_{1}\right)=S\left(\widetilde{\alpha}_{2}\right)$, and when $H\left(\widetilde{\alpha}_{1}\right) \prec H\left(\widetilde{\alpha}_{2}\right)$, then $\widetilde{\alpha}_{1} \prec \widetilde{\alpha}_{2}$; when $H\left(\widetilde{\alpha}_{1}\right)=H\left(\widetilde{\alpha}_{2}\right)$, then $\widetilde{\alpha}_{1}=\widetilde{\alpha}_{2}$

\section{The Proposed Approach for Low-Carbon Suppliers Selection}

5.1. Low-Carbon Suppliers Problem Description. To the low-carbon supplier selection problem in the process of low-carbon building construction projects, for which $S_{i}=$ $\left\{S_{1}, S_{2}, \ldots, S_{m}\right\}(m \geq 2)$ is a discrete and feasible alternative solution set of low-carbon suppliers, $C_{j}=\left\{C_{1}\right.$, $\left.C_{2}, \ldots, C_{n}\right\}(n \geq 2)$ is the finite set of criteria for low-carbon supplier selection in the process of low-carbon building construction projects; $w=\left(w_{1}, w_{2}, \ldots, w_{n}\right)^{T}$ is a weight vector, which satisfies $0 \leq w_{j} \leq 1, \sum_{j=1}^{n} w_{j}=1$; and $\eta\left(t_{k}\right)=$ $\left(\eta\left(t_{1}\right), \eta\left(t_{2}\right), \ldots, \eta\left(t_{\psi}\right)\right)^{T}$ is the time weight vector, where $0 \leq \eta\left(t_{k}\right) \leq 1$ and $\sum_{k=1}^{\psi} \eta\left(t_{k}\right)=1$. The value of criteria $C_{j}$, to which solution $S_{i}$ is subject at moment $t_{k}$, is denoted as $X_{i j}\left(t_{k}\right)$, which is subject to an interval-valued triangular intuitionistic fuzzy, denoted as $X_{i j}\left(t_{k}\right) \sim \eta_{i j}\left(t_{k}\right)=$ $\left(\left[a_{i j}\left(t_{k}\right), b_{i j}\left(t_{k}\right), c_{i j}\left(t_{k}\right)\right],\left[d_{i j}\left(t_{k}\right), e_{i j}\left(t_{k}\right), f_{i j}\left(t_{k}\right)\right]\right)$, forming a matrix $D_{X_{i j}\left(t_{k}\right)}=\left(\left[a_{i j}\left(t_{k}\right), b_{i j}\left(t_{k}\right), c_{i j}\left(t_{k}\right)\right],\left[d_{i j}\left(t_{k}\right), e_{i j}\left(t_{k}\right)\right.\right.$, $\left.\left.f_{i j}\left(t_{k}\right)\right]\right)_{m \times n}$ based on $\psi$ moment of criteria for low-carbon supplier selection in the process of low-carbon building construction projects. Low-carbon supplier selection problems consist of multiple dimensions, such as supplier, criteria, and time. Integration operator and determining time sequence weight are important technologies to reduce dimensionality and solve low-carbon supplier selection problem under interval-valued intuitionistic fuzzy environment.

\subsection{Interval-Valued Triangular Fuzzy Number Intuitionistic} Fuzzy Bonferroni Means Operator

Definition 6. Let $p, q \geq 0$, and $a_{i}(i=1,2, \ldots, n)$ be a collection of nonnegative numbers [48]. If

$$
B^{p, q}\left(a_{1}, a_{2}, \ldots, a_{n}\right)=\left(\frac{1}{n(n-1)} \sum_{\substack{i, j=1 \\ i \neq j}}^{n} a_{i}^{p} a_{j}^{q}\right)^{1 / p+q},
$$

then, $B^{p, q}$ is called the Bonferroni mean (BM).

Definition 7. Let $\widetilde{\alpha}_{i}=\left(\left[a_{i}, b_{i}, c_{i}\right],\left[d_{i}, e_{i}, f_{i}\right]\right)$ as a collection of IVTFNIFNs. For any $p, q>0$, if 
$\operatorname{IVTFNIFBM}^{p, q}\left(\widetilde{\alpha}_{1}, \widetilde{\alpha}_{2}, \ldots, \widetilde{\alpha}_{n}\right)=\left(\frac{1}{n(n-1)}\left(\underset{\substack{i, j=1 \\ i \neq j}}{\overbrace{i=1}^{\oplus}}\left(\widetilde{\alpha}_{i}^{p} \otimes \widetilde{\alpha}_{j}^{q}\right)\right)\right)^{1 / p+q}$
Theorem 1. Let $p, q>0$, and $\widetilde{\alpha}_{i}=\left(\left[a_{i}, b_{i}, c_{i}\right],\left[d_{i}, e_{i}, f_{i}\right]\right)$ as a collection of positive IVTFNIFN. Then, by using the IVTFNIFBM, is also an IVTFNIFN, and

$$
\begin{aligned}
& \operatorname{IVTFNIFBM}^{p, q}\left(\widetilde{\alpha}_{1}, \widetilde{\alpha}_{2}, \ldots, \widetilde{\alpha}_{n}\right)=([a, b, c],[d, e, f]), \\
& a=\left(1-\prod_{\substack{i, j=1 \\
i \neq j}}^{n}\left(1-a_{i}^{p} a_{j}^{q}\right)^{1 / n(n-1)}\right)^{1 / p+q}, \\
& b=\left(1-\prod_{\substack{i, j=1 \\
i \neq j}}^{n}\left(1-b_{i}^{p} b_{j}^{q}\right)^{1 / n(n-1)}\right)^{1 / p+q}, \\
& c=\left(1-\prod_{\substack{i, j=1 \\
i \neq j}}^{n}\left(1-c_{i}^{p} c_{j}^{q}\right)^{1 / n(n-1)}\right)^{1 / p+q}, \\
& d=1-\left(1-\prod_{\substack{i, j=1 \\
i \neq j}}^{n}\left(1-\left(1-d_{i}\right)^{p}\left(1-d_{j}\right)^{q}\right)^{1 / n(n-1)}\right)^{1 / p+q}, \\
& e=1-\left(1-\prod_{\substack{i, j=1 \\
i \neq j}}^{n}\left(1-\left(1-e_{i}\right)^{p}\left(1-e_{j}\right)^{q}\right)^{1 / n(n-1)}\right)^{1 / p+q}, \\
& f=1-\left(1-\prod_{\substack{i, j=1 \\
i \neq j}}^{n}\left(1-\left(1-f_{i}\right)^{p}\left(1-f_{j}\right)^{q}\right)^{1 / n(n-1)}\right)^{1 / p+q} .
\end{aligned}
$$

Proof. By operations (4) and (6), we have

$$
\begin{aligned}
& \alpha_{i}^{p}=\left(\left[a_{i}^{p}, b_{i}^{p}, c_{i}^{p}\right],\left[1-\left(1-d_{i}\right)^{p}, 1-\left(1-e_{i}\right)^{p}, 1-\left(1-f_{i}\right)^{p}\right]\right), \\
& \alpha_{j}^{q}=\left(\left[a_{j}^{q}, b_{j}^{q}, c_{j}^{q}\right],\left[1-\left(1-d_{j}\right)^{q}, 1-\left(1-e_{j}\right)^{q}, 1-\left(1-f_{j}\right)^{q}\right]\right),
\end{aligned}
$$

and then

$$
\begin{aligned}
\alpha_{i}^{p} \otimes \alpha_{j}^{q}= & \left(\left[a_{i}^{p} a_{j}^{q}, b_{i}^{p} b_{j}^{q}, c_{i}^{p} c_{j}^{q}\right],\left[1-\left(1-d_{i}\right)^{p}\left(1-d_{j}\right)^{q},\right.\right. \\
& \left.\left.1-\left(1-e_{i}\right)^{p}\left(1-e_{j}\right)^{q}, 1-\left(1-f_{i}\right)^{p}\left(1-f_{j}\right)^{q}\right]\right) .
\end{aligned}
$$

As following, we first prove that

$$
\begin{aligned}
\underset{\substack{i, j=1 \\
i \neq j}}{n}\left(\alpha_{i}^{p} \otimes \alpha_{j}^{q}\right)= & \left(\left[\left(1-\prod_{\substack{i, j=1 \\
i \neq j}}^{n}\left(1-a_{i}^{p} a_{j}^{q}\right)\right),\left(1-\prod_{\substack{i, j=1 \\
i \neq j}}^{n}\left(1-b_{i}^{p} b_{j}^{q}\right)\right),\left(1-\prod_{\substack{i, j=1 \\
i \neq j}}^{n}\left(1-c_{i}^{p} c_{j}^{q}\right)\right)\right],\right. \\
& {\left[\prod_{\substack{i, j=1 \\
i \neq j}}^{n}\left(1-\left(1-d_{i}\right)^{p}\left(1-d_{j}\right)^{q}\right), \prod_{\substack{i, j=1 \\
i \neq j}}^{n}\left(1-\left(1-e_{i}\right)^{p}\left(1-e_{j}\right)^{q}\right), \prod_{\substack{i, j=1 \\
i \neq j}}^{n}\left(1-\left(1-f_{i}\right)^{p}\left(1-f_{j}\right)^{q}\right)\right], }
\end{aligned}
$$




$$
\begin{aligned}
\underset{\substack{\oplus \\
i, j=1 \\
i \neq j}}{2}\left(\widetilde{\alpha}_{i}^{p} \otimes \widetilde{\alpha}_{j}^{q}\right)= & \left(\widetilde{\alpha}_{1}^{p} \otimes \widetilde{\alpha}_{2}^{q}\right) \oplus\left(\widetilde{\alpha}_{2}^{p} \otimes \widetilde{\alpha}_{1}^{q}\right) \\
= & \left(\left[1-\left(1-a_{1}^{p} a_{2}^{q}\right)\left(1-a_{2}^{p} a_{1}^{q}\right), 1-\left(1-b_{1}^{p} b_{2}^{q}\right)\left(1-b_{2}^{p} b_{1}^{q}\right), 1-\left(1-c_{1}^{p} c_{2}^{q}\right)\left(1-c_{2}^{p} c_{1}^{q}\right)\right]\right), \\
& {\left[\left(1-\left(1-d_{1}\right)^{p}\left(1-d_{2}\right)^{q}\right)\left(1-\left(1-d_{2}\right)^{p}\left(1-d_{1}\right)^{q}\right),\left(1-\left(1-e_{1}\right)^{p}\left(1-e_{2}\right)^{q}\right)\left(1-\left(1-e_{2}\right)^{p}\left(1-e_{1}\right)^{q}\right),\right.} \\
& \left.\left.\left(1-\left(1-f_{1}\right)^{p}\left(1-f_{2}\right)^{q}\right)\left(1-\left(1-f_{2}\right)^{p}\left(1-f_{1}\right)^{q}\right)\right]\right) .
\end{aligned}
$$

(2) If (14) holds for $n=k$, i.e., then, when $n=k+1$, we have

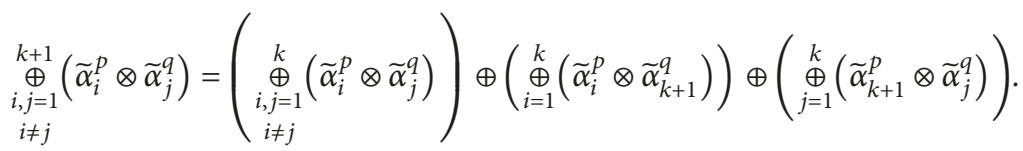

Now, we prove that

$$
\begin{aligned}
& \underset{i=1}{\stackrel{\oplus}{(}}\left(\widetilde{\alpha}_{i}^{p} \otimes \widetilde{\alpha}_{k+1}^{q}\right)=\left(\left[1-\prod_{i=1}^{k}\left(1-a_{i}^{p} a_{k+1}^{q}\right), 1-\prod_{i=1}^{k}\left(1-b_{i}^{p} b_{k+1}^{q}\right), 1-\prod_{i=1}^{k}\left(1-c_{i}^{p} c_{k+1}^{q}\right)\right],\right. \\
& \left.\left[\prod_{i=1}^{k}\left(1-\left(1-d_{i}\right)^{p}\left(1-d_{k+1}\right)^{q}\right), \prod_{i=1}^{k}\left(1-\left(1-e_{i}\right)^{p}\left(1-e_{k+1}\right)^{q}\right), \prod_{i=1}^{k}\left(1-\left(1-f_{i}\right)^{p}\left(1-f_{k+1}\right)^{q}\right)\right]\right)
\end{aligned}
$$

by using mathematical induction on $k$ as follows.

(1) For $k=2$, then by (17), we have

$$
\begin{aligned}
& \widetilde{\alpha}_{i}^{p} \otimes \widetilde{\alpha}_{2+1}^{q}=\left(\left[a_{i}^{p} a_{2+1}^{q}, b_{i}^{p} b_{2+1}^{q}, c_{i}^{p} c_{2+1}^{q}\right],\left[1-\left(1-d_{i}\right)^{p}\left(1-d_{2+1}\right)^{q}, 1-\left(1-e_{i}\right)^{p}\left(1-e_{2+1}\right)^{q}, 1-\left(1-f_{i}\right)^{p}\left(1-f_{2+1}\right)^{q}\right]\right) \quad i=1,2, \\
& \text { and thus } \\
& \underset{i=1}{\stackrel{\oplus}{(}}\left(\widetilde{\alpha}_{i}^{p} \otimes \widetilde{\alpha}_{2+1}^{q}\right)=\left(\widetilde{\alpha}_{1}^{p} \otimes \widetilde{\alpha}_{2+1}^{q}\right) \oplus\left(\widetilde{\alpha}_{2}^{p} \otimes \widetilde{\alpha}_{2+1}^{q}\right) \\
& =\left(\left[1-\left(1-a_{1}^{p} a_{2+1}^{q}\right)\left(1-a_{2}^{p} a_{2+1}^{q}\right), 1-\left(1-b_{1}^{p} b_{2+1}^{q}\right)\left(1-b_{2}^{p} b_{2+1}^{q}\right), 1-\left(1-c_{1}^{p} c_{2+1}^{q}\right)\left(1-c_{2}^{p} c_{2+1}^{q}\right)\right],\right. \\
& {\left[\left(1-\left(1-d_{1}\right)^{p}\left(1-d_{2+1}\right)^{q}\right)\left(1-\left(1-d_{2}\right)^{p}\left(1-d_{2+1}\right)^{q}\right),\left(1-\left(1-e_{1}\right)^{p}\left(1-e_{2+1}\right)^{q}\right)\left(1-\left(1-e_{2}\right)^{p}\left(1-e_{2+1}\right)^{q}\right),\right.} \\
& \left.\left.\left(1-\left(1-f_{1}\right)^{p}\left(1-f_{2+1}\right)^{q}\right)\left(1-\left(1-f_{2}\right)^{p}\left(1-f_{2+1}\right)^{q}\right)\right]\right) .
\end{aligned}
$$

(2) If (17) holds for $k=k_{0}$, i.e., then, when $k=k_{0}+1$, we have 


$$
\begin{aligned}
\underset{i=1}{k_{0}+1}\left(\widetilde{\alpha}_{i}^{p} \otimes \widetilde{\alpha}_{k_{0}+2}^{q}\right)= & \stackrel{k}{i=1}_{i=1}^{\oplus}\left(\widetilde{\alpha}_{i}^{p} \otimes \widetilde{\alpha}_{k_{0}+1}^{q}\right) \oplus\left(\widetilde{\alpha}_{k_{0}+1}^{p} \otimes \widetilde{\alpha}_{k_{0}+2}^{q}\right) \\
= & \left(\left[1-\left(\prod_{i=1}^{k_{0}}\left(1-a_{i}^{p} a_{k_{0}+1}^{q}\right)\right)\left(1-a_{k_{0}+1}^{p} a_{k_{0}+2}^{q}\right), 1-\left(\prod_{i=1}^{k_{0}}\left(1-b_{i}^{p} b_{k_{0}+1}^{q}\right)\right)\left(1-b_{k_{0}+1}^{p} b_{k_{0}+2}^{q}\right), 1-\left(\prod_{i=1}^{k_{0}}\left(1-c_{i}^{p} c_{k_{0}+1}^{q}\right)\right)\left(1-c_{k_{0}+1}^{p} c_{k_{0}+2}^{q}\right)\right]\right. \\
& {\left[\prod_{i=1}^{k_{0}}\left(1-\left(1-d_{i}\right)^{p}\left(1-d_{k_{0}+1}\right)^{q}\right)\left(1-\left(1-d_{k_{0}+1}\right)^{p}\left(1-d_{k_{0}+2}\right)^{q}\right)\right.} \\
& \prod_{i=1}^{k_{0}}\left(1-\left(1-e_{i}\right)^{p}\left(1-e_{k_{0}+1}\right)^{q}\right)\left(1-\left(1-e_{k_{0}+1}\right)^{p}\left(1-e_{k_{0}+2}\right)^{q}\right) \\
& \left.\left.\prod_{i=1}^{k_{0}}\left(1-\left(1-f_{i}\right)^{p}\left(1-f_{k_{0}+1}\right)^{q}\right)\left(1-\left(1-f_{k_{0}+1}\right)^{p}\left(1-f_{k_{0}+2}\right)^{q}\right)\right]\right) \\
= & \left(\left[1-\prod_{i=1}^{k_{0}+1}\left(1-a_{i}^{p} a_{k+1}^{q}\right), 1-\prod_{i=1}^{k_{0}+1}\left(1-b_{i}^{p} b_{k+1}^{q}\right), 1-\prod_{i=1}^{k_{0}+1}\left(1-c_{i}^{p} c_{k+1}^{q}\right)\right]\right. \\
& {\left.\left[\prod_{i=1}^{k_{0}+1}\left(1-\left(1-d_{i}\right)^{p}\left(1-d_{k+1}\right)^{q}\right), \prod_{i=1}^{k_{0}+1}\left(1-\left(1-e_{i}\right)^{p}\left(1-e_{k+1}\right)^{q}\right), \prod_{i=1}^{k_{0}+1}\left(1-\left(1-f_{i}\right)^{p}\left(1-f_{k+1}\right)^{q}\right)\right]\right) }
\end{aligned}
$$

i.e., (17) holds for $k=k_{0}+1$; thus, (17) holds for all $k$.

$$
\begin{aligned}
\underset{j=1}{\oplus}\left(\widetilde{\alpha}_{k+1}^{p} \otimes \widetilde{\alpha}_{j}^{q}\right)= & \left(\left[1-\prod_{j=1}^{k}\left(1-a_{k+1}^{p} a_{j}^{q}\right), 1-\prod_{j=1}^{k}\left(1-b_{k+1}^{p} b_{j}^{q}\right), 1-\prod_{j=1}^{k}\left(1-c_{k+1}^{p} c_{j}^{q}\right)\right],\right. \\
& {\left.\left[\prod_{j=1}^{k}\left(1-\left(1-d_{k+1}\right)^{p}\left(1-d_{j}\right)^{q}\right), \prod_{j=1}^{k}\left(1-\left(1-e_{k+1}\right)^{p}\left(1-e_{j}\right)^{q}\right), \prod_{j=1}^{k}\left(1-\left(1-f_{k+1}\right)^{p}\left(1-f_{j}\right)^{q}\right)\right]\right) . }
\end{aligned}
$$

Thus, by (16), (17), and (21), we further transform (16) as

$$
\begin{aligned}
& \underset{\substack{i, j=1 \\
i \neq j}}{\oplus+1}\left(\widetilde{\alpha}_{i}^{p} \otimes \widetilde{\alpha}_{j}^{q}\right)=\left(\underset{\substack{\oplus \\
i, j=1 \\
i \neq j}}{\underset{i \neq j}{i}}\left(\widetilde{\alpha}_{i}^{p} \otimes \widetilde{\alpha}_{j}^{q}\right)\right) \oplus\left(\underset{\substack{\oplus \\
i=1}}{k}\left(\widetilde{\alpha}_{i}^{p} \otimes \widetilde{\alpha}_{k+1}^{q}\right)\right) \oplus\left(\underset{\substack{\oplus \\
j=1}}{k}\left(\widetilde{\alpha}_{k+1}^{p} \otimes \widetilde{\alpha}_{j}^{q}\right)\right) \\
& =\left(\left[1-\prod_{\substack{i, j=1 \\
i \neq j}}^{k}\left(1-a_{i}^{p} a_{j}^{q}\right), 1-\prod_{\substack{i, j=1 \\
i \neq j}}^{k}\left(1-b_{i}^{p} b_{j}^{q}\right), 1-\prod_{\substack{i, j=1 \\
i \neq j}}^{k}\left(1-c_{i}^{p} c_{j}^{q}\right)\right],\right. \\
& \left.\left[\prod_{\substack{i, j=1 \\
i \neq j}}^{k}\left(1-\left(1-d_{i}\right)^{p}\left(1-d_{j}\right)^{q}\right), \prod_{\substack{i, j=1 \\
i \neq j}}^{k}\left(1-\left(1-e_{i}\right)^{p}\left(1-e_{j}\right)^{q}\right), \prod_{\substack{i, j=1 \\
i \neq j}}^{k}\left(1-\left(1-f_{i}\right)^{p}\left(1-f_{j}\right)^{q}\right)\right]\right) \\
& \oplus\left(\left[1-\left(\prod_{i=1}^{k_{0}+1}\left(1-a_{i}^{p} a_{k+1}^{q}\right)\right), 1-\left(\prod_{i=1}^{k_{0}+1}\left(1-b_{i}^{p} b_{k+1}^{q}\right)\right), 1-\left(\prod_{i=1}^{k_{0}+1}\left(1-c_{i}^{p} c_{k+1}^{q}\right)\right)\right],\right. \\
& {\left[\prod_{i=1}^{k_{0}+1}\left(1-\left(1-d_{i}\right)^{p}\left(1-d_{k+1}\right)^{q}\right), \prod_{i=1}^{k_{0}+1}\left(1-\left(1-e_{i}\right)^{p}\left(1-e_{k+1}\right)^{q}\right),\right.} \\
& \left.\left.\prod_{i=1}^{k_{0}+1}\left(1-\left(1-f_{i}\right)^{p}\left(1-f_{k+1}\right)^{q}\right)\right]\right) \oplus\left(\left[1-\prod_{j=1}^{k}\left(1-a_{\mathrm{k}+1}^{p} a_{j}^{q}\right), 1-\prod_{j=1}^{k}\left(1-b_{\mathrm{k}+1}^{p} b_{j}^{q}\right), 1-\prod_{j=1}^{k}\left(1-c_{k+1}^{p} c_{j}^{q}\right)\right],\right. \\
& \left.\left[\prod_{j=1}^{k}\left(1-\left(1-d_{\mathrm{k}+1}\right)^{p}\left(1-d_{j}\right)^{q}\right), \prod_{j=1}^{k}\left(1-\left(1-e_{k+1}\right)^{p}\left(1-e_{j}\right)^{q}\right), \prod_{j=1}^{k}\left(1-\left(1-f_{k+1}\right)^{p}\left(1-f_{j}\right)^{q}\right)\right]\right) \\
& =\left(\left[1-\left(\prod_{\substack{i, j=1 \\
i \neq j}}^{k+1}\left(1-a_{i}^{p} a_{j}^{q}\right)\right), 1-\left(\prod_{\substack{i, j=1 \\
i \neq j}}^{k+1}\left(1-b_{i}^{p} b_{j}^{q}\right)\right), 1-\left(\prod_{\substack{i, j=1 \\
i \neq j}}^{k+1}\left(1-c_{i}^{p} c_{j}^{q}\right)\right)\right],\right. \\
& {\left[\prod_{\substack{i, j=1 \\
i \neq j}}^{k+1}\left(1-\left(1-d_{i}\right)^{p}\left(1-d_{j}\right)^{q}\right), \prod_{\substack{i, j=1 \\
i \neq j}}^{k+1}\left(1-\left(1-e_{i}\right)^{p}\left(1-e_{j}\right)^{q}\right), \prod_{\substack{i, j=1 \\
i \neq j}}^{k+1}\left(1-\left(1-f_{i}\right)^{p}\left(1-f_{j}\right)^{q}\right)\right],}
\end{aligned}
$$




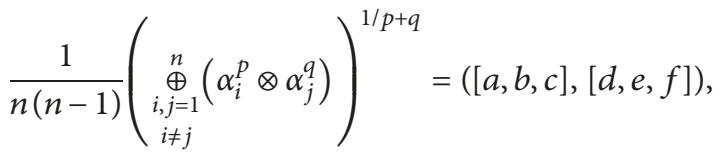

$$
\begin{aligned}
& a=\left(1-\prod_{\substack{i, j=1 \\
i \neq j}}^{n}\left(1-a_{i}^{p} a_{j}^{q}\right)^{1 / n(n-1)}\right)^{1 / p+q}, \\
& b=\left(1-\prod_{\substack{i, j=1 \\
i \neq j}}^{n}\left(1-b_{i}^{p} b_{j}^{q}\right)^{1 / n(n-1)}\right)^{1 / p+q}, \\
& c=\left(1-\prod_{\substack{i, j=1 \\
i \neq j}}^{n}\left(1-c_{i}^{p} c_{j}^{q}\right)^{1 / n(n-1)}\right)^{1 / p+q}, \\
& d=1-\left(1-\prod_{\substack{i, j=1 \\
i \neq j}}^{n}\left(1-\left(1-d_{i}\right)^{p}\left(1-d_{j}\right)^{q}\right)^{1 / n(n-1)}\right)^{1 / p+q}, \\
& e=1-\left(1-\prod_{\substack{i, j=1 \\
i \neq j}}^{n}\left(1-\left(1-e_{i}\right)^{p}\left(1-e_{j}\right)^{q}\right)^{1 / n(n-1)}\right)^{1 / p+q}, \\
& f=1-\left(1-\prod_{\substack{i, j=1 \\
i \neq j}}^{n}\left(1-\left(1-f_{i}\right)^{p}\left(1-f_{j}\right)^{q}\right)^{1 / n(n-1)}\right)^{1 / p+q},
\end{aligned}
$$

which completes the proof of Theorem 1 .

Based on the studies above, we can look at some properties of IVTFNIFBM as below:

(1) Idempotency: if $\widetilde{\alpha}_{i}=\left(\left[a_{i}, b_{i}, c_{i}\right],\left[d_{i}, e_{i}, f_{i}\right]\right)=\widetilde{\alpha}=$ $([a, b, c],[d, e, f])$, for all $i$, then

$\operatorname{IVTFNIFN}^{p, q}\left(\widetilde{\alpha}_{1}, \widetilde{\alpha}_{2}, \ldots, \widetilde{\alpha}_{n}\right)=\operatorname{IVTFNIFN}^{p, q}(\widetilde{\alpha}, \widetilde{\alpha}, \ldots, \widetilde{\alpha})=\widetilde{\alpha}$.

(2) Commutativity: let $\left(\widetilde{\alpha}_{1}, \widetilde{\alpha}_{2}, \ldots, \widetilde{\alpha}_{n}\right)$ as a positive collection of IVTFNIFN, then

$\operatorname{IVTFNIFN}^{p, q}\left(\widetilde{\alpha}_{1}, \widetilde{\alpha}_{2}, \ldots, \widetilde{\alpha}_{n}\right)=\operatorname{IVTFNIFN}^{p, q}\left(\widetilde{\alpha}_{1}, \widetilde{\alpha}_{2}, \ldots, \widetilde{\alpha}_{n}\right)$,

where $\left(\widetilde{\alpha}_{1}, \widetilde{\alpha}_{2}, \ldots, \widetilde{\alpha}_{n}\right)$ is any permutation of $\left(\widetilde{\alpha}_{1}, \widetilde{\alpha}_{2}, \ldots, \widetilde{\alpha}_{n}\right)$.

(3) Monotonicity: let $\tilde{\alpha}_{i}=\left(\left[a_{i}, b_{i}, c_{i}\right],\left[d_{i}, e_{i}, f_{i}\right]\right)(i=$ $1,2, \ldots, n)$ and $\widetilde{\alpha}_{i}^{\Delta}=\left(\left[\widehat{\alpha}_{i}, \widehat{b}_{i}, \widehat{c}_{i}\right],\left[\widehat{d}_{i}, \widehat{e}_{i}, \widehat{f}_{i}\right]\right)$ are two positive collections of IVTFNIFN; if, $a_{i} \geq \widehat{\alpha}_{i}, b_{i} \geq$ $\hat{b}_{i}, c_{i} \geq \widehat{c}_{i}, d_{i} \leq \widehat{d}_{i}, e_{i} \leq \widehat{e}_{i}, f_{i} \leq \widehat{f}_{i}$, for all $i$, then
$\operatorname{IVTFNIFN}^{p, q}\left(\widetilde{\alpha}_{1}, \widetilde{\alpha}_{2}, \ldots, \widetilde{\alpha}_{n}\right) \geq \operatorname{IVTFNIFN}^{p, q}\left(\widetilde{\alpha}_{1}^{\Delta}, \widetilde{\alpha}_{2}^{\Delta}, \ldots, \widetilde{\alpha}_{n}^{\Delta}\right)$.

(4) Boundedness: let $\widetilde{\alpha}_{i}=\left(\left[a_{i}, b_{i}, c_{i}\right],\left[d_{i}, e_{i}, f_{i}\right]\right)(i=1$, $2, \ldots, n)$ as a positive collection of IVTFNIFN, then

$$
\begin{aligned}
& \widetilde{\alpha}^{-} \leq \operatorname{IVTFNIFBM}^{p, q}\left(\widetilde{\alpha}_{1}, \widetilde{\alpha}_{2}, \ldots, \widetilde{\alpha}_{n}\right) \leq \widetilde{\alpha}^{+}, \\
& \widetilde{\alpha}^{-}=\left(\left[\min _{i} a_{i}, \min _{i} b_{i}, \min _{i} c_{i}\right],\left[\min _{i} d_{i}, \min _{i} e_{i}, \min _{i} f_{i}\right]\right), \\
& \widetilde{\alpha}^{+}=\left(\left[\max _{i} a_{i}, \max _{i} b_{i}, \max _{i} c_{i}\right],\left[\max _{i} d_{i}, \max _{i} e_{i}, \max _{i} f_{i}\right]\right) .
\end{aligned}
$$

The TFNIFWBM considers the interaction between criteria for low-carbon supplier selection in the process of low-carbon building construction projects, but they have different levels of importance in low-carbon supplier selection. Therefore, we first propose IVTFNIFBM operator. 
In the aforementioned analysis, we consider the attribute interrelationships, which are important. However, in many practical situations, we should take into account the weights of the data. So, we first define an IVTFNIFBM operator.
Definition 8. Let $\widetilde{\alpha}_{i}=\left(\left[a_{i}, b_{i}, c_{i}\right],\left[d_{i}, e_{i}, f_{i}\right]\right)$ as a positive collection of IVTFNIFN. $w=\left(w_{1}, w_{2}, \ldots, w_{n}\right)^{T}$ is the weight vector of $\tilde{\alpha}_{i}(i=1,2, \ldots, n)$, where $w_{i} \geq 0$ and $\sum_{i=1}^{n} w_{i}=1$. If

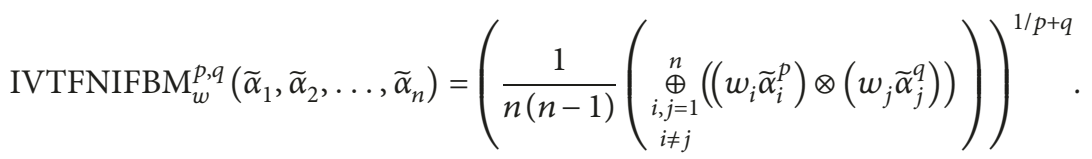

Then IVTFNIFN ${ }_{w}^{p, q}$ is called the interval-valued triangular fuzzy number intuitionistic fuzzy weighted Bonferroni mean (IVTFNIFWBM).

Similar to Theorem 1, we have Theorem 2.

Theorem 2. Let $\tilde{\alpha}_{i}=\left(\left[a_{i}, b_{i}, c_{i}\right],\left[d_{i}, e_{i}, f_{i}\right]\right)(i=1,2, \ldots, n)$ be a positive collection of IVTFNIFN. $w=\left(w_{1}, w_{2}, \ldots, w_{n}\right)^{T}$ is the weight vector of $\widetilde{\alpha}_{i}(i=1,2, \ldots, n)$, where $w_{i} \geq 0$ and $\sum_{i=1}^{n} w_{i}=1$. Then, the aggregated value, by using the IVTFNIFWBM, is also an IVTFNIFN, and

$$
\operatorname{IVTFNIFWBM}^{p, q}\left(\widetilde{\alpha}_{1}, \widetilde{\alpha}_{2}, \ldots, \widetilde{\alpha}_{n}\right)=([\hat{a}, \widehat{b}, \hat{c}],[\hat{d}, \hat{e}, \hat{f}]) .
$$

$$
\begin{aligned}
& \text { Then } \\
& \widehat{a}=\left(1-\prod_{\substack{i, j=1 \\
i \neq j}}^{n}\left(1-\left(w_{i} a_{i}\right)^{p}\left(w_{j} a_{j}\right)^{q}\right)^{1 / n(n-1)}\right)^{1 / p+q}, \\
& \widehat{b}=\left(1-\prod_{\substack{i, j=1 \\
i \neq j}}^{n}\left(1-\left(w_{i} b_{i}\right)^{p}\left(w_{j} b_{j}\right)^{q}\right)^{1 / n(n-1)}\right)^{1 / p+q}, \\
& \widehat{c}=\left(1-\prod_{\substack{i, j=1 \\
i \neq j}}^{n}\left(1-\left(w_{i} c_{i}\right)^{p}\left(w_{j} c_{j}\right)^{q}\right)^{1 / n(n-1)}\right)^{1 / p+q}, \\
& \widehat{d}=1-\left(1-\prod_{\substack{i, j=1 \\
i \neq j}}^{n}\left(1-\left(1-w_{i} d_{i}\right)^{p}\left(1-w_{j} d_{j}\right)^{q}\right)^{1 / n(n-1)}\right)^{1 / p+q}, \\
& \widehat{e}=1-\left(1-\prod_{\substack{i, j=1 \\
i \neq j}}^{n}\left(1-\left(1-w_{i} e_{i}\right)^{p}\left(1-w_{j} e_{j}\right)^{q}\right)^{1 / n(n-1)}\right)^{1 / p+q}, \\
& \widehat{f}=1-\left(1-\prod_{\substack{i, j=1 \\
i \neq j}}^{n}\left(1-\left(1-w_{i} f_{i}\right)^{p}\left(1-w_{j} f_{j}\right)^{q}\right)^{1 / n(n-1)}\right)^{1 / p+q} .
\end{aligned}
$$

Theorem 2 can be proved by mathematical induction; in a similar way, we can prove that IVTFNIFWBM operator also has idempotency, commutativity, monotonicity, and boundedness features, and the detailed proof procedures are omitted here.

\subsection{Time Weight Based on Time Degree and Ideal Solution}

Definition 9. Supposing $\eta\left(t_{k}\right)=\left(\eta\left(t_{1}\right), \eta\left(t_{2}\right), \ldots, \eta\left(t_{k}\right)\right)^{T}$ represents time sequence weight vector, where $\eta\left(t_{k}\right)$ represents the weight of kth time period, and $\eta\left(t_{k}\right) \in[0,1]$, $\sum_{k=1}^{\psi} \eta\left(t_{k}\right)=1$, time sequence weight indicates the attentionattaching degree on different time periods in decisionmaking process.

Information entropy can reflect the uptake degree of time weight vector against information quantity; the greater the entropy is, the less the information quantity it contains. Therefore, based on maximum entropy principle, we solve the time weight of time degree and information entropy and set up a nonlinear programming model as follows:

$$
\begin{cases}\max & I=-\sum_{k=1}^{\psi} \eta\left(t_{k}\right) \ln \eta\left(t_{k}\right), \\ \text { s.t. } & \lambda=\sum_{k=1}^{\psi} \frac{\psi-k}{\psi-1} \eta\left(t_{k}\right), \sum_{k=1}^{\psi} \eta\left(t_{k}\right)=1, \eta\left(t_{k}\right) \in[0,1] .\end{cases}
$$

When $\lambda$ gets closer to 0 , indicating decision-maker attaches more preference to recent information of time series; when $\lambda$ gets closer to 1 , indicating decision-maker attaches more preference to forward information of time series. We solve this model by Lingo.11 software and acquire the time sequence weight vector.

Definition 10. Based on the Definition 8, when we consider the equilibrium of time preference of decision makers in different time periods, we can determine time weight based on an objective function of maximization closeness degree to ideal solution with subjective time preference. We denote $\eta\left(t_{k}\right)^{+}$as positive ideal time weight, and negative ideal time weight is denoted by $\eta\left(t_{k}\right)^{-}$.

Let the distance between the two time weight vectors $\eta\left(\tilde{t_{k}}\right)=\left(\eta\left(\widetilde{t_{1}}\right), \eta\left(\widetilde{t_{2}}\right), \ldots, \eta\left(\widetilde{t_{\psi}}\right)\right)^{T}$ and $\eta\left(\widehat{t_{k}}\right)=\left(\eta\left(\widehat{t_{1}}\right), \eta\left(\widehat{t_{2}}\right), \ldots\right.$, $\left.\eta\left(\widetilde{t_{\psi}}\right)\right)^{T}$ be

$$
d\left(\eta\left(\tilde{t_{k}}\right), \eta\left(\widehat{t_{k}}\right)\right)=\sqrt{\sum_{k=1}^{\psi}\left(\eta\left(\widetilde{t_{k}}\right)-\eta\left(\widehat{t_{k}}\right)\right)^{2}}
$$

Then the distances between a time weight vector $\eta\left(t_{k}\right)=$ $\left(\eta\left(t_{1}\right), \eta\left(t_{2}\right), \ldots, \eta\left(t_{k}\right)\right)^{T}$ and positive and negative ideal time weight vectors respectively are 


$$
\begin{aligned}
& d\left(\eta\left(t_{k}\right), \eta\left(t_{k}\right)^{+}\right)=\sqrt{\sum_{k=1}^{\psi-1} \eta\left(t_{k}\right)^{2}+\left(1-\eta\left(t_{\psi}\right)\right)^{2}}, \\
& d\left(\eta\left(t_{k}\right), \eta\left(t_{k}\right)^{-}\right)=\sqrt{\left(1-\eta\left(t_{1}\right)\right)^{2}+\sum_{k=2}^{\psi} \eta\left(t_{k}\right)^{2}} .
\end{aligned}
$$

$$
\begin{cases}\max & c\left(\eta\left(t_{k}\right), \eta\left(t_{k}\right)^{+}\right)=\frac{\sqrt{\left(1-\eta\left(t_{1}\right)\right)^{2}+\sum_{k=2}^{\psi} \eta\left(t_{k}\right)^{2}}}{\sqrt{\left(1-\eta\left(t_{1}\right)\right)^{2}+\sum_{k=2}^{\psi} \eta\left(t_{k}\right)^{2}}+\sqrt{\sum_{k=1}^{\psi-1} \eta\left(t_{k}\right)^{2}+\left(1-\eta\left(t_{\psi}\right)\right)^{2}}} \\ \text { s.t. } & \lambda=\sum_{k=1}^{\psi} \frac{\psi-k}{\psi-1} \eta\left(t_{k}\right), \sum_{k=1}^{\psi} \eta\left(t_{k}\right)=1, \eta\left(t_{k}\right) \in[0,1], \quad k=1,2, \ldots, \psi\end{cases}
$$

Based on the thought of "stress the present rather than the past", the more recent information can fully reflect the characteristics of decision-making attributes, and it would be more effective for decision-making evaluation results. We solve this model by Lingo.11 software and acquire the time sequence weight vector.
The relative closeness degree between time weight vector $\eta\left(t_{k}\right)$ and ideal time weight vector $\eta\left(t_{k}\right)^{+}$can be obtained:

$$
c\left(\eta\left(t_{k}\right), \eta\left(t_{k}\right)^{+}\right)=\frac{d\left(\eta\left(t_{k}\right), \eta\left(t_{k}\right)^{-}\right)}{d\left(\eta\left(t_{k}\right), \eta\left(t_{k}\right)^{+}\right)+d\left(\eta\left(t_{k}\right), \eta\left(t_{k}\right)^{-}\right)} .
$$

Then, based on time degree and ideal solution, constructing a nonlinear programming model is as follows:

$$
\begin{cases}\max & R=l \frac{\sqrt{\left(1-\eta\left(t_{1}\right)\right)^{2}+\sum_{k=2}^{\psi} \eta\left(t_{k}\right)^{2}}}{\sqrt{\left(1-\eta\left(t_{1}\right)\right)^{2}+\sum_{k=2}^{\psi} \eta\left(t_{k}\right)^{2}}+\sqrt{\sum_{k=1}^{\psi-1} \eta\left(t_{k}\right)^{2}+\left(1-\eta\left(t_{\psi}\right)\right)^{2}}}+(1-l)\left(-\sum_{k=1}^{\psi} \eta\left(t_{k}\right) \ln \eta\left(t_{k}\right)\right), \\ \text { s.t. } & \lambda=\sum_{k=1}^{\psi} \frac{\psi-k}{\psi-1} \eta\left(t_{k}\right), \sum_{k=1}^{\psi} \eta\left(t_{k}\right)=1, \eta\left(t_{k}\right) \in[0,1], \quad k=1,2, \ldots, \psi,\end{cases}
$$

where $l$ is an adjustable coefficient, $l \in[0,1]$, if $l$ is close to 0 , it is the sign that decision makers are more inclined to the time weight based on objective information-driven, and when the $l$ is close to 1 , the decision makers are more emphasized on time weight based on subjective preference information. We also solve this model by Lingo.11 software and acquire the time sequence weight vector.

\subsection{The Weight of Attribute Based on Entropy-TOPSIS}

Definition 11. Let $\widetilde{\alpha}=\left(a_{i}, b_{i}, c_{i}\right)$ and $\widetilde{\beta}=\left(\widehat{a}_{i}, \widehat{b}_{i}, \widehat{c}_{i}\right)$ are two collections of IVTFNIFNs, then the distances between $\widetilde{\alpha}$ and $\widetilde{\beta}$ is

$$
D(\widetilde{\alpha}, \widetilde{\beta})=\sqrt{\frac{1}{3}\left[\left(a_{i}-\widehat{a}_{i}\right)^{2}+\left(b_{i}-\widehat{b}_{i}\right)^{2}+\left(c_{i}-\widehat{c}_{i}\right)^{2}\right]} .
$$

Based on the decision-making situations of uncertainty, multicriteria, and finite case, we let. $\widetilde{A}=\left(A_{1}, A_{2}, \ldots, A_{n}\right)$ as $n$ alternatives, $\widetilde{C}=\left(C_{1}, C_{2}, \ldots, C_{n}\right)$ as a collection of attributes. $w=\left(w_{1}, w_{2}, \ldots, w_{n}\right)^{T}$ is the weight vector of $\widetilde{C}$, where $w_{i} \geq 0$ and $\sum_{i=1}^{n} w_{i}=1$.
Based on (31) and (35), this paper constructs a comprehensive time weight while considering the uptake ability of time weight against information as well as the effectiveness of recent decision-making information, as follows:
If the performance of the alternative $A_{i}$ with respect to the attributes $C_{j}$ is measured by an IVTFNIFNs, all IVTFNIFNs are contained in an intuitionistic fuzzy decision $D=\left(\widetilde{d_{i j}}\right)_{n \times m}$.

Definition 12. If $d_{j}^{*}=\left(\tilde{d}_{j}, \widehat{d}_{j}, \hat{d}_{j}\right)$ is an ideal performance values of attributes, there are, generally, benefit criteria and cost criteria.

When the performance values of the benefit type, then $\widetilde{d}_{j}=\max \widetilde{d}_{i j}, \widehat{d}_{j}=\max \widehat{d}_{i j}, \widehat{d}_{j}=\max \widehat{d}_{i j}$.

When the performance values of the cost type, then $\tilde{d}_{j}=\min _{i} \tilde{d}_{i j}, \widehat{d}_{j}=\min \widehat{d}_{i j}, \hat{d}_{j}=\min _{i} \widehat{d}_{i j}$.

In general, the alternatives have a small difference in the performance value of attributes, namely, the attributes have a small influence on a multiple attribute decision-making problem. Conversely, the more the difference, the more the effect. Therefore, the larger the performance value of attributes deviation, the larger the attributes weight. We can learn from the entropy that the lower the entropy is, the more the information quantity it contains.

Due to the limit of entropy, we need to acquire standardized decision-making matrix by (37) and get a distance between the attributes and the ideal attributes. 
Steps of the weight of attribute are provided as follows:

First, constructing the ideal performance values of attributes by Definition 11 based on the IVTFNIFN. Next, calculating the distance from each attributes and ideal attributes via Formula (37). Then, we can construct a distance matrix $R_{1}=\left(\tilde{r}_{i j}\right)_{n \times m}, \quad R_{2}=\left(\widehat{r}_{i j}\right)_{n \times m}$ where we use $r_{i j}^{*}=r_{i j} / \sum_{i=1}^{m} r_{i j}$, then conduct standardization on IVTFNIFN distance matrix $R_{1}^{*}=\left(\widetilde{r}_{i j}^{*}\right)_{n \times m}$ and $R_{2}^{*}=\left(\hat{r}_{i j}^{*}\right)_{n \times m}$. Finally, calculating the weight of attributes $\widetilde{C}=\left(C_{1}, C_{2}, \ldots, C_{n}\right)$.

$$
\begin{aligned}
& e_{j}=-\sum_{i=1}^{n}\left(r_{i j}^{*} \times \ln r_{i j}^{*}\right) / \ln n, \\
& w_{j}^{1}=\frac{1-e_{j}^{1}}{\sum_{j=1}^{m}\left(1-e_{j}^{1}\right)}(j=1,2, \ldots, m), \\
& w_{j}^{2}=\frac{1-e_{j}^{2}}{\sum_{j=1}^{m}\left(1-e_{j}^{2}\right)}(j=1,2, \ldots, m), \\
& w_{j}=\sqrt{w_{j}^{1} w_{j}^{2}}(j=1,2, \ldots, m) .
\end{aligned}
$$

We can know the final comprehensive weight, $w_{j}(j=1,2,3, \ldots, m)$.

5.5. Steps of Low-Carbon Supplier Selection in the Process of Low-Carbon Building Construction Projects. According to the calculation process of the above model for lowcarbon supplier selection in the process of low-carbon building construction projects, the calculation steps are as follows.

Step 1. The original information matrix $D_{X_{i j}\left(t_{k}\right)}=\left(\left[a_{i j}\left(t_{k}\right)\right.\right.$, $\left.\left.b_{i j}\left(t_{k}\right), c_{i j}\left(t_{k}\right)\right],\left[d_{i j}\left(t_{k}\right), e_{i j}\left(t_{k}\right), f_{i j}\left(t_{k}\right)\right]\right)_{m \times n}$ of low-carbon supplier selection is given by our project collaborators who are construction project managers, practitioners, and industry experts, based on the different $\psi$ moments.

Step 2. Based on the main criteria of low-carbon supplier selection for constructor in Table 1, we form information matrix for criteria and calculate the criteria weight set $w=$ $\left(w_{1}, w_{2}, \ldots, w_{n}\right)^{T}$ according to Formulas (37) and (38). Then, we calculate the time sequence weight set $\eta\left(t_{k}\right)=$ $\left(\eta\left(t_{1}\right), \eta\left(t_{2}\right), \ldots, \eta\left(t_{\psi}\right)\right)^{T}$ according to Formula (36) by solving the model via Lingo 11.0 software.

Step 3. Utilizing the IVTFIFWBM operator to aggregate the criteria information of low-carbon supplier selection based on the criteria weight which is calculated in Step 2. Then, we need to aggregate all individual criteria information $C_{j}$ potential low-carbon suppliers into a collective criteria information matrix $D_{X_{i j}\left(t_{k}\right)}^{\prime}=\left(\left[a_{i j}^{\prime}\left(t_{k}\right)\right.\right.$, $\left.\left.b_{i j}^{\prime}\left(t_{k}\right), c_{i j}^{\prime}\left(t_{k}\right)\right], \quad\left[d_{i j}^{\prime}\left(t_{k}\right), e_{i j}^{\prime}\left(t_{k}\right), f_{i j}^{\prime}\left(t_{k}\right)\right]\right)_{m \times n}$ according to Formula (23).

Step 4. Gathering the information of time dimension of lowcarbon supplier selection based on the time sequence weight set $\eta\left(t_{k}\right)=\left(\eta\left(t_{1}\right), \eta\left(t_{2}\right), \ldots, \eta\left(t_{\psi}\right)\right)^{T}$. Then, we create the comprehensive decision information matrix $D_{X_{i}}^{\prime \prime}=\left(\left[a_{i}^{\prime \prime}\right.\right.$, $\left.\left.b_{i}^{\prime \prime}, c_{i}^{\prime \prime}\right],\left[d_{i}^{\prime \prime}, e_{i}^{\prime \prime}, f_{i}^{\prime \prime}\right]\right)_{m \times 1}$ via Formula (29) for the single dimension to potential low-carbon suppliers $S_{i}$.

Step 5. Finally, selecting the best low-carbon supplier in the process of low-carbon building construction projects based on ranking value $L_{i}=\left(L_{1}, L_{2}, \ldots, L_{i}\right)$ and further determining the priority sequence of low-carbon supplier $S_{i}(i=1,2, \ldots, m)$.

\section{Case Study}

6.1. Case Company Background. According to the above analysis, the proposed method is applied on the case of the housing construction project entity in the construction industry to solve low-carbon supplier selection problem.

Company HFG, founded in 1986, is a builder enterprise, which has special qualifications for construction, located in Tai Yuan, a city of Shan Xi Province in China. HFG's business scope involves housing construction general contracting, infrastructure construction, real estate investment, engineering design, and other fields in the major cities. HFG will be committed to green housing technology development and practice, with product innovation and the provision of low-carbon building products as the development goal. For builder HFG, one of the important issues is how to reduce carbon emissions of construction projects to enhance lowcarbon competitiveness and profit. In this circumstances, $H F G$ needs to select its low-carbon supplier from a large number of suppliers in the process of low-carbon building construction projects.

As builder $H F G$ has some experience accumulation in the supplier selection, it is still a difficult problem for $H F G$ to select its best low-carbon supplier from these potential suppliers in the process of low-carbon building construction projects. On the one hand, builder $H$ has established a criterion, which is not appropriate to use it to select low-carbon supplier. It did not establish the criteria for the low-carbon supplier selection in the process of low-carbon building construction projects. On the other hand, builder HFG not only has to nondimensionalize the criteria to previous supplier selection, but also more focus on the single period of decision criteria information, and even if $H F G$ considers multiple timings during supplier selection, it may still lead to subjectivity and objectivity in the time weight. Moreover, the selection method is very difficult for builder $H F G$ to deal with qualitative criteria in the process of low-carbon supplier selection.

6.2. Application of the Proposed Criteria and Method. To builder $H F G$, the proposed criteria and method is suitable to be used to select low-carbon supplier in the process of low-carbon building construction projects, because the managers and practitioner's understanding of the weights of criteria for low-carbon supplier selection is in the fuzzy state in builder HFG. In addition, expert scoring method which is usually used to select traditional supplier in their 
TABLE 2: Original evaluation criteria information matrix at the moment $t_{1}$.

\begin{tabular}{cccccc}
\hline & $C_{1}$ & $C_{2}$ & $C_{3}$ & $C_{4}$ & $C_{5}$ \\
\hline \multirow{2}{*}{$S_{1}$} & $([0.6,0.7,0.8]$, & $([0.5,0.6,0.6]$, & $([0.1,0.1,0.4]$, & $([0.6,0.7,0.7]$, & $([0.7,0.8,0.9]$, \\
& $[0.1,0.2,0.3])$ & $[0.2,0.2,0.2])$ & $[0.2,0.2,0.5])$ & $[0.1,0.2,0.3])$ & $(0.1,0.1,0.1])$ \\
$S_{2}$ & $([0.2,0.3,0.4]$, & $([0.4,0.4,0.5]$, & $([0.1,0.2,0.2]$, & $([0.3,0.4,0.5]$, & $([0.5,0.6,0.7]$, \\
& $[0.4,0.5,0.5])$ & $[0.4,0.4,0.5])$ & $[0.6,0.7,0.8])$ & $[0.2,0.2,0.3])$ & $(0.2,0.2,0.3])$ \\
$S_{3}$ & $([0.4,0.5,0.6]$, & $([0.3,0.4,0.5]$, & $([0.2,0.3,0.4]$, & $([0.6,0.7,0.8]$, & $([0.7,0.7,0.7]$, \\
& $[0.1,0.2,0.2])$ & $[0.3,0.4,0.4])$ & $[0.5,0.6,0.6])$ & $[0.1,0.2,0.2])$ & $(0.1,0.2,0.2])$ \\
$S_{4}$ & $([0.6,0.6,0.7]$, & $([0.4,0.5,0.6]$, & $([0.6,0.6,0.7]$, & $([0.4,0.5,0.5]$, & $([0.2,0.3,0.4]$, \\
& $[0.2,0.2,0.2])$ & $[0.2,0.2,0.3])$ & $[0.1,0.1,0.1])$ & $[0.1,0.2,0.3])$ & $[0.5,0.6,0.6])$ \\
\hline
\end{tabular}

construction projects makes the proposed criteria and method more realistic and practical.

For the moment, builder $H F G$ is required to purchase a batch of rebar for a low-carbon building in Tai Yuan. After the primary selection of steel production enterprises, there are four enterprises $S_{i}=\left\{S_{1}, S_{2}, S_{3}, S_{4}\right\}$ to enter the final selection. Builder $H$ needs to select its steel supplier from 4 main low-carbon suppliers by the proposed criteria and method. Therefore, H's 15 managers, practitioners, and experts are asked to determine the criteria of low-carbon supplier selection to construction projects based on the preliminary list of criteria compiled including literature review about low-carbon supplier selection and the builder $H$ 's actual situation. It can be seen in Table 1 including 5 main criteria and 17 subcriteria. Moreover, they select the time sequence set of different historical periods for nearly three years, $t_{k}=\left(t_{1}, t_{2}, t_{3}\right)$, for the previously mentioned potential low-carbon suppliers. For the sake of simplicity, we only give out the calculation for the 5 main criteria. The evaluated values of 4 main suppliers which are given by 15 managers, practitioners, and experts are listed in Tables 2-4.

Based on the original evaluation criteria information matrix of low-carbon supplier selection which only includes supplier $S_{1}$, supplier $S_{2}$, supplier $S_{3}$, and supplier $S_{4}$ in the process of low-carbon building construction projects, per Step 2, according to Formulas (37) and (38), the criteria weight is shown in Table 5. The time degree parameter $\lambda=0.3$, and the discrete time weight vector is solved via Step 2 and Lingo 11.0 software. $\eta\left(t_{k}\right)=\left(\eta\left(t_{1}\right), \eta\left(t_{2}\right)\right.$, $\left.\eta\left(t_{3}\right)\right)^{T}=(0.582,0.236,0.182)$.

Based on the criteria weight vector $w=\left(w_{1}, w_{2}, w_{3}\right.$, $w_{4}, w_{5}$ ), per Step 3 , the five criteria were assembled into a collective the criteria information of low-carbon supplier selection matrix from 3 periods of time. Comprehensive criteria making information of each potential low-carbon suppliers were assembled from different moment, per Step 4 , forming comprehensive selection information matrix for the target single dimension; in the end, the value of each potentiallow-carbon suppliers for the construction project was determined based on Step 5 and is shown in Tables 6-8.

Thus, the low-carbon supplier, who will provide the batch of rebar for the low-carbon building in Tai Yuan, is determined to $S_{1}$. Based on the evaluation and selection above, supplier $S_{1}$ is recommended as builder $H F G$ 's best low-carbon supplier. In fact, builder $H F G$ has given priority to supplier $S_{1}$, who provides the batch of rebar for the lowcarbon building in Tai Yuan, according to the results. In addition, supplier $S_{3}$ is recommended as the reserved lowcarbon supplier. HFG's low-carbon housing technology development and practice will improve its competitiveness and profitability in the construction industry based on the concept of continuous improvement.

\section{Conclusions}

There has been broad consensus on carbon emissions reduction around the world. Low-carbon building not only can bring a healthier and more comfortable living environment, but also can reduce carbon emissions in the construction industry. For constructors, using low-carbon building materials for construction and sustainable development of the environment is particularly important. In addition, GSCM has become an inevitable choice for constructors to cope with the pressure from the government and the market. Therefore, it is one of the most important factors to select low-carbon supplier in the process of lowcarbon building construction projects.

In this paper, we propose a dynamic multiattribute decision-making approach with interval-valued triangular fuzzy numbers intuitionistic fuzzy for low-carbon supplier selection in the process of low-carbon building construction projects. According to the demand of constructors in the process of low-carbon building construction projects, 5 main criteria and 17 subcriteria are established for lowcarbon supplier selection in the construction industry. The proposed method considers interaction between criteria of low-carbon supplier selection and the influence of constructors' subjective preference and objective criteria information. The evaluated values of potential low-carbon suppliers are given by managers, practitioners, and experts. The proposed criteria and method are suitable to use to select low-carbon supplier in the process of low-carbon building construction projects because the managers and practitioner's understanding of the weights of criteria for lowcarbon supplier selection are in the intuitionistic fuzzy due to the nature of unquantifiable and incomplete information in low-carbon supplier selection. In addition, expert scoring method, which is usually used to select traditional supplier in their construction projects, makes the proposed criteria and method more realistic and practical. The proposed criteria and method have been successfully implemented in a case construction project to select the best low-carbon supplier. It not only is much easier for constructors to select low-carbon supplier, but also can make the localization of low-carbon 
TABLE 3: Original evaluation criteria information matrix at the moment $t_{2}$.

\begin{tabular}{cccccc}
\hline & $C_{1}$ & $C_{2}$ & $C_{3}$ & $C_{4}$ & $C_{5}$ \\
\hline \multirow{2}{*}{$S_{1}$} & $([0.2,0.3,0.4]$, & $([0.5,0.6,0.7]$, & $([0.5,0.5,0.6]$, & $([0.6,0.7,0.7]$, & $([0.4,0.5,0.6]$, \\
& $[0.3,0.4,0.4])$ & $[0.2,0.2,0.2])$ & $[0.1,0.2,0.5])$ & $[0.1,0.2,0.3])$ & $(0.1,0.1,0.1])$ \\
$S_{2}$ & $([0.3,0.4,0.5]$, & $([0.4,0.5,0.5]$, & $([0.3,0.4,0.5]$, & $([0.4,0.5,0.6]$, & $([0.6,0.6,0.7]$, \\
& $[0.1,0.2,0.3])$ & $[0.1,0.2,0.2])$ & $[0.1,0.2,0.3])$ & $[0.2,0.2,0.3])$ & $(0.1,0.2,0.2])$ \\
$S_{3}$ & $([0.4,0.5,0.6]$, & $([0.7,0.7,0.7]$, & $([0.4,0.5,0.5]$, & $([0.6,0.7,0.8]$, & $([0.3,0.4,0.5]$, \\
& $[0.1,0.2,0.2])$ & $[0.1,0.1,0.1])$ & $[0.1,0.2,0.3])$ & $[0.1,0.2,0.2])$ & $(0.2,0.3,0.3])$ \\
$S_{4}$ & $([0.6,0.6,0.7]$, & $([0.4,0.5,0.6]$, & $([0.6,0.6,0.7]$, & $([0.2,0.3,0.3]$, & $([0.2,0.3,0.4]$, \\
& $[0.1,0.1,0.1])$ & $[0.2,0.2,0.3])$ & $[0.1,0.1,0.1])$ & $[0.3,0.4,0.5])$ & $[0.5,0.6,0.6])$ \\
\hline
\end{tabular}

TABLE 4: Original evaluation criteria information matrix at the moment $t_{3}$.

\begin{tabular}{cccccc}
\hline & $C_{1}$ & $C_{2}$ & $C_{3}$ & $C_{4}$ & $C_{5}$ \\
\hline \multirow{2}{*}{$S_{1}$} & $([0.6,0.7,0.8]$, & $([0.8,0.9,0.9]$, & $([0.2,0.3,0.4]$, & $([0.7,0.8,0.9]$, & $([0.4,0.5,0.6]$, \\
& $[0.1,0.2,0.2])$ & $[0.1,0.1,0.1])$ & $[0.3,0.4,0.5])$ & $[0.1,0.1,0.1])$ & $(0.2,0.3,0.4])$ \\
$S_{2}$ & $([0.8,0.8,0.9]$, & $([0.7,0.7,0.8]$, & $([0.7,0.7,0.8]$, & $([0.4,0.5,0.6]$, & $([0.5,0.6,0.7]$, \\
& $[0.1,0.1,0.1])$ & $[0.2,0.2,0.2])$ & $[0.1,0.2,0.2])$ & $[0.2,0.2,0.3])$ & $(0.1,0.2,0.2])$ \\
$S_{3}$ & $([0.4,0.5,0.6]$, & $([0.4,0.5,0.5]$, & $([0.4,0.5,0.5]$, & $([0.7,0.7,0.8]$, & $([0.4,0.6,0.7]$, \\
& $[0.1,0.2,0.2])$ & $[0.2,0.3,0.4])$ & $[0.1,0.2,0.3])$ & $[0.1,0.1,0.1])$ & $(0.2,0.3,0.3])$ \\
$S_{4}$ & $([0.3,0.4,0.5]$, & $([0.4,0.5,0.6]$, & $([0.6,0.6,0.7]$, & $([0.4,0.5,0.7]$, & $([0.1,0.2,0.3]$, \\
& $[0.1,0.1,0.3])$ & $[0.2,0.2,0.3])$ & $[0.1,0.1,0.1])$ & $[0.1,0.2,0.3])$ & $[0.5,0.6,0.6])$ \\
\hline
\end{tabular}

TABLE 5: The criteria weight.

\begin{tabular}{cccccc}
\hline & $C_{1}$ & $C_{2}$ & $C_{3}$ & $C_{4}$ & $C_{5}$ \\
\hline$t_{1}$ & 0.199 & 0.192 & 0.208 & 0.201 & 0.200 \\
$t_{2}$ & 0.217 & 0.225 & 0.162 & 0.185 & 0.211 \\
$t_{3}$ & 0.086 & 0.288 & 0.107 & 0.183 & 0.336 \\
\hline
\end{tabular}

TABLE 6: The comprehensive evaluation value under the different $l$.

\begin{tabular}{lc}
\hline & Comprehensive evaluation value \\
\hline$l=0.2$ & $([0.008,0.185,0.213],[0.439,0.452,0.469])$ \\
$S_{1}$ & $([0.007,0.154,0.180],[0.458,0.471,0.482])$ \\
$S_{2}$ & $([0.008,0.177,0.199],[0.444,0.466,0.469])$ \\
$S_{3}$ & $([0.007,0.152,0.180],[0.457,0.465,0.475])$ \\
$S_{4}$ & $([0.008,0.185,0.213],[0.438,0.451,0.468])$ \\
\hline$l=0.5$ & $([0.007,0.153,0.179],[0.458,0.471,0.482])$ \\
$S_{1}$ & $([0.008,0.176,0.197],[0.444,0.466,0.469])$ \\
$S_{2}$ & $([0.007,0.150,0.179],[0.456,0.464,0.475])$ \\
$S_{3}$ & \\
$S_{4}$ & $([0.008,0.181,0.208],[0.436,0.449,0.465])$ \\
\hline$l=0.8$ & $([0.006,0.150,0.175],[0.458,0.470,0.480])$ \\
$S_{1}$ & $([0.007,0.170,0.190],[0.443,0.465,0.468])$ \\
$S_{2}$ & $([0.006,0.144,0.172],[0.453,0.462,0.473])$ \\
$S_{3}$ & \\
$S_{4}$ &
\end{tabular}

supplier more practical and more accurate in the construction industry. Finally, low-carbon supplier selection of builder HFG for a low-carbon building in Tai Yuan is studied to verify the scientificity and feasibility of the proposed criteria and method. The result shows that this criteria and method are of effectiveness and practicality of low-carbon supplier selection in the process of low-carbon building construction projects. Also, it can be mentioned that the
Table 7: The comprehensive evaluation value under the fully objective/subjective information.

\begin{tabular}{lc}
\hline & Comprehensive evaluation value \\
\hline$l=0$ & $([0.008,0.189,0.215],[0.440,0.453,0.466])$ \\
$S_{1}$ & $([0.007,0.169,0.196],[0.444,0.459,0.468])$ \\
$S_{2}$ & $([0.008,0.180,0.200],[0.439,0.461,0.466])$ \\
$S_{3}$ & $([0.006,0.146,0.177],[0.456,0.465,0.477])$ \\
$S_{4}$ & $([0.008,0.174,0.197],[0.436,0.449,0.461])$ \\
\hline$l=1$ & $([0.007,0.158,0.183],[0.437,0.453,0.461])$ \\
$S_{1}$ & $([0.007,0.166,0.184],[0.434,0.455,0.461])$ \\
$S_{2}$ & $([0.006,0.132,0.161],[0.452,0.461,0.473])$ \\
$S_{3}$ & \\
$S_{4}$ &
\end{tabular}

TABLe 8: The value of each potential low-carbon suppliers and the rank results comparison for the construction project.

\begin{tabular}{lcc}
\hline & $S_{i}$ & Ranking result \\
\hline$l=0$ & $(-0.303,-0.322,-0.315,-0.347)$ & $S_{1}>S_{3}>S_{2}>S_{4}$ \\
$l=1$ & $(-0.311,-0.325,-0.321,-0.354)$ & $S_{1}>S_{3}>S_{2}>S_{4}$ \\
$l=0.2$ & $(-0.305,-0.347,-0.321,-0.343)$ & $S_{1}>S_{3}>S_{4}>S_{2}$ \\
$l=0.5$ & $(-0.304,-0.348,-0.322,-0.343)$ & $S_{1}>S_{3}>S_{4}>S_{2}$ \\
$l=0.8$ & $(-0.305,-0.349,-0.326,-0.346)$ & $S_{1}>S_{3}>S_{4}>S_{2}$ \\
\hline
\end{tabular}

proposed model can be easily extended to analyze other management decision problems as a structural model.

This study has some limitations that warrant future research attention. The attribute weight method based on the Entropy-TOPSIS model in this paper is only an objective assignment method. A comprehensive attribute weight method considering the objective assignment information and subjective preferences of decision makers should be studied in the future. Moreover, evaluation criteria information cannot be 
effectively reflected by using IVTFNs under uncertain linguistic environment. For further study, we will extend the proposed method in this paper with linguistic intuitionistic fuzzy number and prospect theory in other civil engineering fields.

\section{Data Availability}

All data generated or analyzed to support the findings of this study are included within the article.

\section{Conflicts of Interest}

The authors declare that they have no conflicts of interest.

\section{Acknowledgments}

The authors would like to thank Min Yan. This research was supported by the National Natural Science Foundation of China (71473055), the Fundamental Research Special Funds for the Central Universities (HEUCFW170912, HEUCFP201824), the Humanities and Social Sciences Foundation of Ministry of Education of China (14YJA630002), the National Natural Science Foundation of China (71804084), the Humanities and Social Sciences Foundation of Ministry of Education of China (15YJC630162), the Humanities and Social Sciences Foundation of Ministry of Education of China (18YJC630245), the China Postdoctoral Science Foundation Funded Project (2017M620814), and the National Social Science Foundation of China (17BGL238).

\section{References}

[1] J. Yudelson, Green Building A to Z: Understanding the Language of Green Building, New Society Publishers, Gabriola Island, BC, Canada, 2007.

[2] IPOC, Climate Change 2014 Synthesis Report, Environmental Policy Collection, vol. 27, no. 2, p. 408, 2014.

[3] B. Kang, Y. Hu, Y. Deng, and D. Zhou, "A new methodology of multicriteria decision-making in supplier selection based on Z-numbers," Mathematical Problems in Engineering, vol. 2016, no. 1, pp. 1-17, 2016.

[4] A. H. I. Lee, H. Y. Kang, C. F. Hsu, and H. C. Hung, "A green supplier selection model for high-tech industry," Expert Systems with Applications, vol. 36, no. 4, pp. 7917-7927, 2009.

[5] C. W. Hsu, T. C. Kuo, S. H. Chen, and A. H. Hu, "Using DEMATEL to develop a carbon management model of supplier selection in green supply chain management," Journal of Cleaner Production, vol. 56, no. 10, pp. 164-172, 2013.

[6] D. Kannan and C. J. C. Jabbour, "Selecting green suppliers based on GSCM practices: Using fuzzy TOPSIS applied to a Brazilian electronics company," European Journal of Operational Research, vol. 233, no. 2, pp. 432-447, 2014.

[7] C. W. Tsui and U. P. Wen, "A hybrid multiple criteria group decision-making approach for green supplier selection in the TFT-LCD industry," Mathematical Problems in Engineering, vol. 2014, Article ID 709872, 13 pages, 2014.

[8] O. Gurel, A. Z. Acar, I. Onden, and I. Gumus, "Determinants of the green supplier selection," Procedia-Social and Behavioral Sciences, vol. 181, no. 4, pp. 131-139, 2015.

[9] H. M. W. Chen, S. Y. Chou, Q. D. Luu, and H. K. Yu, “A fuzzy MCDM approach for green supplier selection from the economic and environmental aspects," Mathematical Problems in Engineering, vol. 2016, Article ID 8097386, 10 pages, 2016.

[10] F. Yu, Y. Yang, D. Chang, F. Yu, and Y. Yang, "Carbon footprint based green supplier selection under dynamic environment," Journal of Cleaner Production, vol. 170, no. 8, pp. 880-889, 2017.

[11] K. Govindan and R. Sivakumar, "Green supplier selection and order allocation in a low-carbon paper industry: integrated multi-criteria heterogeneous decision-making and multi-objective linear programming approaches," Annals of Operations Research, vol. 238, no. 3, pp. 243-276, 2016.

[12] Q. Pang, T. Yang, M. Li, and Y. Shen, "A fuzzy-grey multicriteria decision making approach for green supplier selection in low-carbon supply chain," Mathematical Problems in Engineering, vol. 1, pp. 1-9, 2017.

[13] A. P. C. Chan, A. Darko, and E. A. Effah, "Strategies for promoting green building technologies adoption in the construction industry-An international study," Sustainability, vol. 9, no. 6, p. 969, 2017.

[14] K. Govindan, R. Khodaverdi, and A. Jafarian, "A fuzzy multi criteria approach for measuring sustainability performance of a supplier based on triple bottom line approach," Journal of Cleaner Production, vol. 47, no. 47, pp. 345-354, 2013.

[15] S. Yin, B. Li, H. Dong, and Z. Xing, "A new dynamic multicriteria decision-making approach for green supplier selection in construction projects under time sequence," Mathematical Problems in Engineering, vol. 2017, Article ID 7954784, 13 pages, 2017.

[16] S. Darabi and J. Heydari, "An interval- valued hesitant fuzzy ranking method based on group decision analysis for green supplier selection," IFAC-Papers On Line, vol. 49, no. 2, pp. 12-14, 2016.

[17] W. C. Yeh and M. C. Chuang, "Using multi-objective genetic algorithm for partner selection in green supply chain problems," Expert Systems with Applications, vol. 38, no. 4, pp. 4244-4253, 2011.

[18] A. Kumar, V. Jain, S. Kumar, and C. Chandra, "Green supplier selection: a new genetic/immune strategy with industrial application," Enterprise Information Systems, vol. 10, no. 8, pp. 911-943, 2015.

[19] M. Gupta, "Supplier selection using artificial neural network and genetic algorithm," International Journal of Indian Culture and Business Management, vol. 11, no. 4, pp. 457-472, 2015.

[20] M. Punniyamoorthy, P. Mathiyalagan, and P. Parthiban, “A strategic model using structural equation modeling and fuzzy logic in supplier selection," Expert Systems with Applications, vol. 38, no. 1, pp. 458-474, 2011.

[21] A. Fallahpour, E. U. Olugu, S. N. Musa, D. Khezrimotlagh, and K. Y. Wong, "An integrated model for green supplier selection under fuzzy environment: application of data envelopment analysis and genetic programming approach," Neural Computing and Applications, vol. 27, no. 3, pp. 707725, 2016.

[22] L. H. Li, J. C. Hang, Y. Gao, and C. Y. Mu, "Using an integrated group decision method Based on SVM, TFN-RSAHP, and TOPSIS-CD for cloud service supplier selection," Mathematical Problems in Engineering, vol. 3, pp. 1-14, 2017.

[23] Z. Hu, C. Rao, Y. Zheng, and D. Huang, "Optimization decision of supplier selection in green procurement under the mode of low carbon economy," International Journal of Computational Intelligence Systems, vol. 8, no. 3, pp. 407-421, 2015. 
[24] J. Qin, X. Liu, and W. Pedrycz, "An extended TODIM multicriteria group decision making method for green supplier selection in interval type-2 fuzzy environment," European Journal of Operational Research, vol. 258, no. 4, pp. 626-638, 2017.

[25] P. Ghadimi, A. Dargi, and C. Heavey, "Sustainable supplier performance scoring using audition check-list based fuzzy inference system: a case application in automotive spare part industry," Computers and Industrial Engineering, vol. 105, no. 2, pp. 12-27, 2017.

[26] R. R. Yager, "On generalized Bonferroni mean operators for multi-criteria aggregation," International Journal of Approximate Reasoning, vol. 50, no. 8, pp. 1279-1286, 2009.

[27] Z. S. Xu, "Intuitionistic fuzzy Bonferroni means," IEEE Transactions on Systems, Man, and Cybernetics-Part B: Cybernetics, vol. 41, no. 2, pp. 568-578, 2011.

[28] M. Xia, Z. Xu, and B. Zhu, "Generalized intuitionistic fuzzy Bonferroni means," International Journal of Intelligent Systems, vol. 27, no. 1, pp. 23-47, 2011.

[29] W. Zhou and J. M. He, "Intuitionistic fuzzy geometric Bonferroni means and their application in multicriteria decision making," International Journal of Intelligent Systems, vol. 27, no. 12, pp. 995-1019, 2012.

[30] B. Zhu, "Hesitant fuzzy geometric Bonferroni means," Information Science, vol. 205, no. 1, pp. 72-85, 2012.

[31] P. D. Liu, L. L. Zhang, X. Liu, and P. Wang, "Multi-valued neutrosophic number Bonferroni mean operators with their applications in multiple attribute group decision making," International Journal of Information Technology \& Decision Making, vol. 15, no. 5, pp. 1181-1210, 2016.

[32] X. Liu, Z. Tao, H. Chen, and L. Zhou, "A new interval-valued 2-tuple linguistic bonferroni mean operator and its application to multi-attribute group decision making," International Journal of Fuzzy Systems, vol. 19, no. 1, pp. 86-108, 2017.

[33] G. W. Wei, "Some geometric aggregation functions and their application to dynamic multiple attribute decision making in the intuitionistic fuzzy setting," International Journal of Uncertainty, Fuzziness and Knowledge-Based Systems, vol. 17, no. 2, pp. 179-196, 2011.

[34] J. H. Park, H. J. Cho, and Y. C. Kwun, "Extension of the VIKOR method to dynamic intuitionistic fuzzy multiple attribute decision making," Computers and Mathematics with Applications, vol. 65, no. 4, pp. 731-744, 2013.

[35] S. Yin, B. Li, H. Dong, and Z. Xing, "A new dynamic multicriteria decision making approach for green supplier selection in construction projects under time sequence," Mathematical problems in Engineering, vol. 2, pp. 1-13, 2017.

[36] Z. S. Xu, "On multi-period multi-attribute decision making," Knowledge-Based Systems, vol. 21, no. 2, pp. 164-171, 2008.

[37] Z. S. Xu and J. Chen, "Binomial distribution based approach to deriving time series weights," in IEEE International Conference on Industrial Engineering and Engineering Management, pp. 154-158, Bangkok, Thailand, 2007.

[38] P. Liu and F. Teng, "Multiple criteria decision making method based on normal interval-valued intuitionistic fuzzy generalized aggregation operator," Complexity, vol. 21, no. 5, pp. 277-290, 2016.

[39] R. Sadiq and S. Tesfamariam, "Probability density functions based weights for ordered weighted averaging (OWA) operators: an example of water quality indices," European Journal of Operational Research, vol. 183, no. 3, pp. 1350-1365, 2007.
[40] X. Z. Zhang and C. X. Zhu, "Generalized precedence order method with ranking preference for multi-attribute decision making," Systems Engineering-Theory and Practice, vol. 33, no. 11, pp. 2852-2858, 2013.

[41] B. Sun and X. F. Xu, "A dynamic stochastic decision-making method based on discrete time sequences," Knowledge-Based Systems, vol. 105, no. 4, pp. 23-28, 2016.

[42] G. W. Wei, "Two-tuple linguistic multiple attribute group decision making with incomplete attribute weight information," Systems Engineering and Electronics, vol. 30, no. 2, pp. 273-277, 2008.

[43] Z. S. Chen and Y. S. Li, "Approach for normal triangular fuzzy stochastic multiple attribute decision making based on prospect mean-variance rule," Control and Decision, vol. 29, no. 7, pp. 1239-1249, 2014.

[44] Y. M. Wang, C. P. Que, and Y. X. Lan, "Hesitant fuzzy TOPSIS multi-attribute decision method based on prospect theory," Control and Decision, vol. 32, no. 5, pp. 864-870, 2017.

[45] K. T. Atanassov, "Intuitionistic fuzzy sets," Fuzzy Sets and Systems, vol. 20, pp. 87-96, 1986.

[46] Z. S. Xu and Q. Chen, "A multi-attribute decision-making process based on interval-valued intuitionistic fuzzy Bonferroni mean," Journal of Systems Engineering and Electronics, vol. 20, no. 2, pp. 217-228, 2011.

[47] X. F. Wang, "Fuzzy number intuitionistic fuzzy geometric aggregation operators and their application to decision making," Control and Decision, vol. 23, no. 6, pp. 607-612, 2008.

[48] C. Bonferroni, "Sulle medie multiple di potenze," Bolletino Matematica Italiana, vol. 5, pp. 267-270, 1950. 


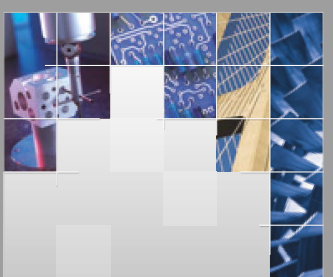

\section{Enfincering}
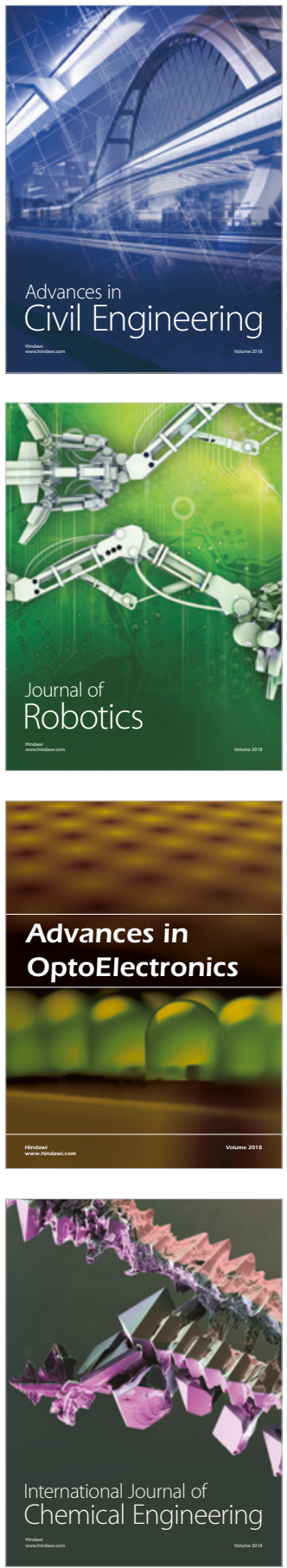

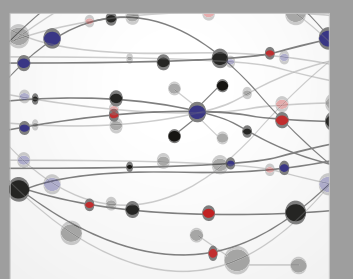

\section{Rotating \\ Machinery}

The Scientific World Journal

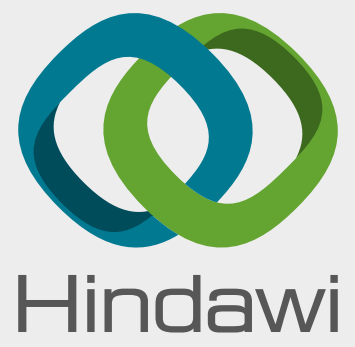

Submit your manuscripts at

www.hindawi.com
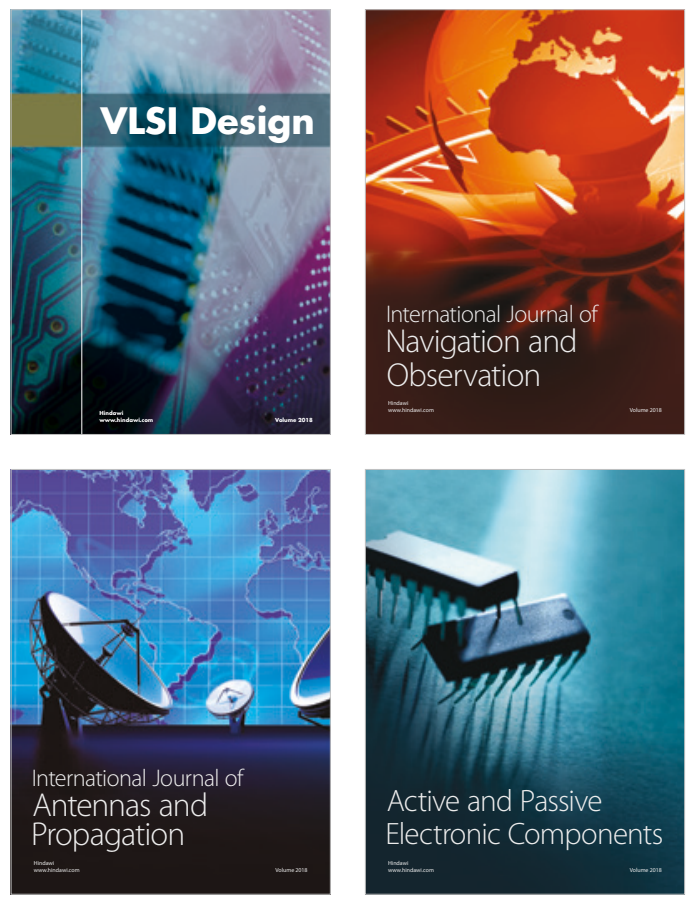
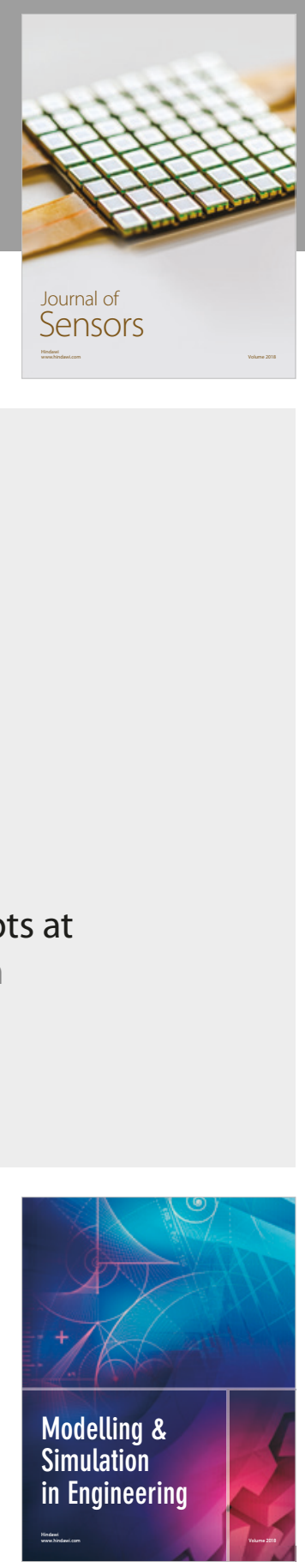

\section{Advances \\ Multimedia}
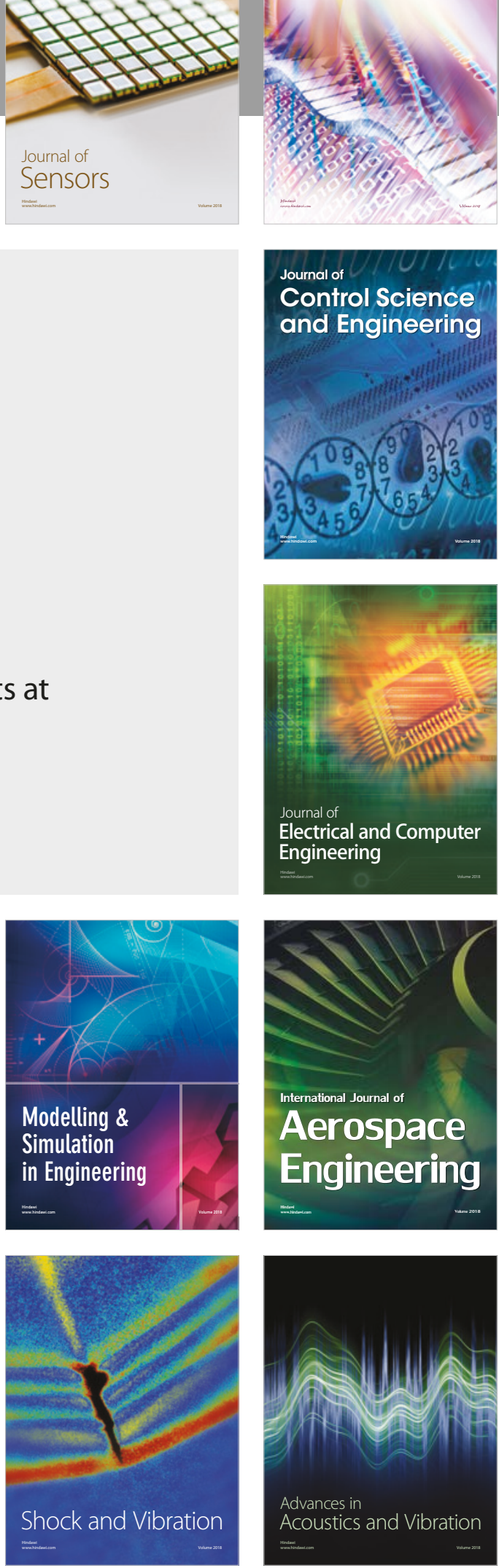